bioRxiv preprint doi: https://doi.org/10.1101/2021.04.18.440348; this version posted April 19, 2021. The copyright holder for this preprint

(which was not certified by peer review) is the author/funder, who has granted bioRxiv a license to display the preprint in perpetuity. It is made available under aCC-BY-NC-ND 4.0 International license.

\title{
1 Chemical phylogenetics of the staphylococcal quorum sensing landscape
}

3 Bengt H. Gless ${ }^{1}$, Benjamin S. Bejder ${ }^{1}$, Ludovica Vitolo ${ }^{1}$, Leonor Marques ${ }^{2}$, Paal S. Andersen ${ }^{2,3}$,

4 Martin S. Bojer ${ }^{2}$, Hanne Ingmer ${ }^{2}$ and Christian A. Olsen ${ }^{1 *}$

5

$6 \quad{ }^{1}$ Center for Biopharmaceuticals and Department of Drug Design and Pharmacology, Faculty of

7 Health and Medical Sciences, University of Copenhagen, Universitetsparken 2, DK-2100,

8 Copenhagen, Denmark.

$9 \quad{ }^{2}$ Department of Veterinary and Animal Sciences, Faculty of Health and Medical Sciences, University

10 of Copenhagen, Stigbøjlen 4, DK-1870 Frederiksberg C, Denmark.

$11{ }^{3}$ Department of Bacteria, Parasites and Fungi, Statens Serum Institut, Artillerivej 5, DK-2300

12 Copenhagen, Denmark

13 *Correspondence to: cao@sund.ku.dk

14

15 


\section{Abstract}

18 Staphylococci utilize secreted autoinducing peptides (AIPs) to regulate group behaviour through a

19 process called quorum sensing (QS). Here, we survey the QS interaction landscape within the

20 Staphylococcus genus by assembling a unique compound collection, comprising all the currently

21 known AIPs. These ribosomally synthesized and posttranslationally modified peptides (RiPPs) were

22 obtained by chemical synthesis and mapping of their ability to modulate QS was evaluated using

23 reporter strains of common human and animal colonizing pathogens (S. aureus, S. epidermidis, $S$.

24 lugdunensis). The resulting map of $>200$ native QS interactions provides a holistic view of nodes that contribute to the complex signalling network within the Staphylococcus genus. This overview reveals surprising cross-species QS induction and identify the first pan-inhibitory AIP, which is then shown to attenuate MRSA induced skin infection in a mouse model. Our results expose a complex universe of possible staphylococcal interactions and provide further impetus for development of therapeutics based on QS modulators targeting antibiotic resistant pathogens. 
Staphylococci are common colonizers of humans and animals with a genus currently consisting of 57 species and 26 subspecies ${ }^{1}$. The genus represents some of the most abundant microbes found in the human microbiota, where the bacteria are involved in complex interactions with the host and other bacteria $^{2}$. A prominent example is the pathogenic species Staphylococcus aureus (SA), which is carried asymptomatically by $30 \%$ of the human population causing $76 \%$ of skin and soft-tissue infections, despite being a relatively poor colonizer of the skin $^{3}$. Emerging methicillin-resistant strains of SA (MRSA) are causing pressure on the public health sector ${ }^{4,5}$ and heavily contribute to the threat of a post-antibiotic era ${ }^{6}$. While SA is mainly colonizing the nasal cavity, the human skin is highly populated by coagulase-negative staphylococci $(\mathrm{CoNS})^{3,7,8}$ of which the most abundant species, $S$. epidermidis (SE), can be found on the skin of nearly all humans ${ }^{9}$. Its roles as symbiont are manifold ${ }^{10}$ with recent studies showing a beneficial role for the host ${ }^{11,12}$, while at the same time causing medical device infections ${ }^{9}$. Other CoNS, such as S. hominis, S. haemolyticus and S. lugdunensis (SL), ${ }^{3,7,8}$ are also common commensal bacteria, which contribute to the complexity of interactions that shape the skin microbiome ${ }^{13}$. Quorum sensing (QS) plays an important role in the transition from harmless skin colonizer to invasive pathogen ${ }^{14,15}$ and this is regulated through the secretion and detection of autoinducing peptides (AIPs), containing a thiolactone (lactone for S. intermedius group ${ }^{16}$. The AIPmediated QS machinery is encoded by a chromosomal locus termed accessory gene regulator (agr), which controls the expression of virulence factors, Agr proteins, and AIPs (Extended Data Fig. 1a) ${ }^{14,15}$. Cross-species QS interference in human microbiota has received therapeutic interest as it can repress virulence gene expression in pathogenic staphylococci including Staphylococcus aureus ${ }^{17,18}$. However, the specific interactions that occur between co-inhabiting species have only been sporadically investigated ${ }^{19-24}$. Studies have investigated the AIPs of $\mathrm{SA}^{25-27}$ as well as non-aureus staphylococci ${ }^{20-24}$ as QS inhibitors and a recent pioneering study even investigated the disease outcome of SA infection upon co-colonization with the AIP producing commensal bacterium $S$. hominis in a human clinical trial ${ }^{28}$. Another skin commensal bacterium S. lugdunensis (SL) has proven pathogenic by causing severe endocarditis ${ }^{29}$ and has been targeted in one previous study ${ }^{30}$. 
However, the effect of non-cognate AIPs, except those from S. aureus, on different agr systems, including that of the common skin colonizer S: epidermidis (SE) has received surprisingly limited attention despite its abundance on the human $\operatorname{skin}^{31-33}$. The growing number of identified AIPs and the lack of information about bacterial crosstalk therefore encouraged us to investigate interactions of all known AIPs (1-26; Extended Fig. 2) with SA agr-I-IV, SE agr-I-III, and SL agr-I

\section{Identification of new autoinducing peptides using an improved trapping protocol}

We recently developed a method for the rapid identification of AIPs from bacterial supernatants (see Extended Fig. 3), which led to a substantial increase in the number of known AIPs $^{34}$. The most timeconsuming step in this procedure is the lyophilization of the supernatant. Therefore we optimized the protocol to allow trapping of the AIPs directly from the bacterial supernatant by adjusting the $\mathrm{pH}$ of the supernatant and adding reducing reagent (Supplementary Figs. 1-4) ${ }^{17,35}$. We identified three new AIPs, namely $S$. pasteuri AIP-I (15), S. succinus AIP-I (16), and S. cohnii AIP-I (17) (Fig. 1a, structures (1-26)], originating from 19 species and covering 5 of the 6 phylogenetic species groups as classified trough multi-locus analysis (Extended Data Fig. 2) ${ }^{36}$.

The substantially less time-consuming identification of AIPs also enabled rapid parallel analysis of the agr groups of $46 \mathrm{SE}$ isolates from a collection of 10 human nasal swabs (Fig. 1b, Supplementary Fig. 8-10), furnishing an identification frequency of close to $70 \%$ (31 of 46). The SE agr-I group was the most prevalent variant in the ensemble of isolates (16), followed by agr-II (9) and agr-III (6).

81 None of the analyzed strains secreted AIPs corresponding to the predicted AIP of SE agr-IV, which remains unidentified ${ }^{37}$. Interestingly, several nasal swaps contained more than one agr variant of SE,

83 which was also previously found by PCR analysis of healthy human nasal swabs ${ }^{38}$, underscoring the complex composition of the staphylococcal colonization. 

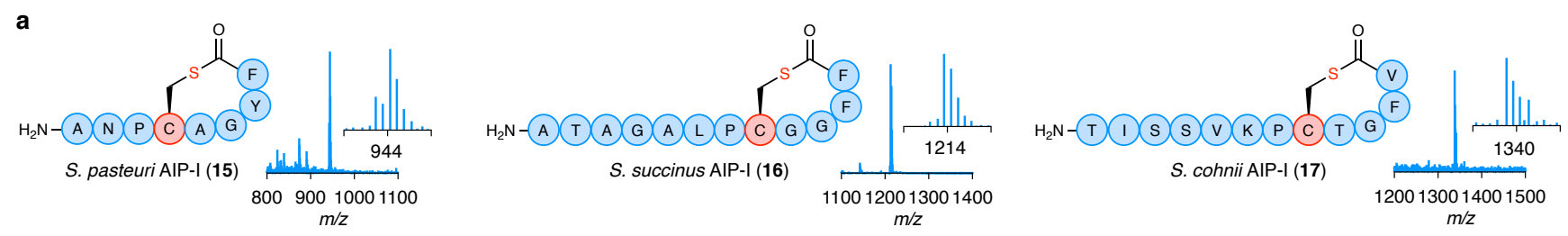

b
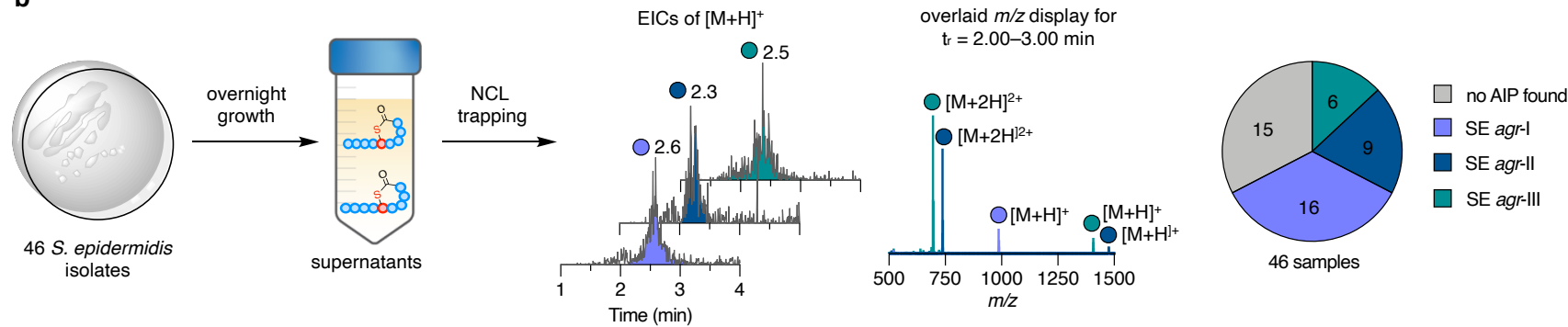

S. epidermidis AIP-I (5); $[\mathrm{M}+\mathrm{H}]^{+} ; m / z=993.4$

S. epidermidis AIP-II (6); [M+H] $]^{+} ; m / z=1475.6$

S. epidermidis AIP-III (7); [M+H]+; $m / z=1416.6$

Fig. 1 | Identification of AIPs through native chemical ligation (NCL) trapping. a, Newly identified AIPs of three CoNS species; S. pasteuri AIP-I (15), S. succinus AIP-I (16), and S. cohnii AIP-I (17). b, NCL trapping performed on bacterial supernatants of 46 isolates of SE from 10 human nasal swabs. The three trapped SeAIPs (5-7) in an analytic LC-MS run eluted between 2.00-3.00 min, as shown for the extracted ion chromatograms (EICs) of $m / z[\mathrm{M}+\mathrm{H}]^{+}\left(\mathrm{t}_{\mathrm{r}}=2.6,2.3\right.$ and $\left.2.5 \mathrm{~min}\right)$. The nasal swab strains were analyzed through combined $\mathrm{m} / \mathrm{z}$ display $\left(\mathrm{t}_{\mathrm{r}}=2.00-3.00 \mathrm{~min}\right)$ and inspection for $[\mathrm{M}+\mathrm{H}]^{+}$and $[\mathrm{M}+2 \mathrm{H}]^{2+}$ of the corresponding trapped $\mathrm{Se}$-AIPs.

The substantially less time-consuming identification of AIPs also enabled rapid parallel analysis of the agr groups of $46 \mathrm{SE}$ isolates from a collection of 10 human nasal swabs (Fig. 1b, Supplementary Fig. 8-10), furnishing an identification frequency of close to $70 \%$ (31 of 46). The SE agr-I group was the most prevalent variant in the ensemble of isolates (16), followed by agr-II (9) and agr-III (6). None of the analyzed strains secreted AIPs corresponding to the predicted AIP of SE agr-IV, which remains unidentified ${ }^{37}$. Interestingly, several nasal swaps contained more than one agr variant of SE, which was also previously found by PCR analysis of healthy human nasal swabs ${ }^{38}$, underscoring the complex composition of the staphylococcal colonization.

\section{Quorum sensing interaction map of all known AIPs}

Having compiled all 26 currently known AIPs (see the supplementary information for synthetic details), we first determined the half maximal inhibitory concentrations $\left(\mathrm{IC}_{50}\right)$ for QS inhibition against SA agr-I-IV of the 12 newly synthesized AIPs in the previously applied $\beta$-lactamase reporter 
strain assay (Extended Data Table 1; Supplementary Fig. 11-14) to compare their potencies to the previously tested $\mathrm{AIPs}^{34}$. However, to include assessment of the QS modulation properties against

110 SE and SL, we turned to fluorescent reporter strains. Thus, screening of the 26 AIPs and Sa-AIP-III

111 D4A (27), a known pan-inhibtior of SA agr-I-IV, ${ }^{26}$ against 8 GFP/YFP-producing reporter strains of

112 SA, SE, and SL provides a comparable data set across all tested strains (Fig 2a, Supplementary Figs. 113 15-20), which correlated well with data points from previously reported QS interference studies 114 (Supplementary Table 1 and 2).

115 The 208 native QS interactions were predominantly inhibitory (161 of 208), with only 27 out of 208 116 (13\%) showing no effect at $1 \mu \mathrm{M}$ (Fig. 2b). The SA agr-I-III variants were highly susceptible to most 117 tested AIPs also representing the majority of interactions reaching $>95 \%$ inhibition at $2.5 \mathrm{nM}$ (19 of 118 24). In agreement with our $\beta$-lactamase data set (Extended Data Table 1), SA agr-IV is significantly 119 less susceptible to inhibition by other AIPs compared to SA agr-I-III and only the synthetic inhibitor 12027 was able to fully suppress $a g r$ activity of that particular strain at $2.5 \mathrm{nM}$. The SE $a g r$-I-III variants 121 were nearly equally susceptible to the inhibition by AIPs of other species, as observed for as SA agr122 I-III, but the majority of inhibitory interactions required higher AIP concentrations to achieve full 123 inhibition. SL agr-I was the least affected by AIPs from other staphylococci with only 4 of 26 124 interactions being fully inhibitory at $50 \mathrm{nM}$. The AIPs of SA (1-4) showed surprisingly little effect 125 against SE agr-I-III and vice versa with the exception of Se-AIP-I (5) for SA agr-III. This stands in 126 contrast to the majority of AIPs, which showed no strong tendency to inhibit only SA or SE but rather 127 showed similar inhibition profiles against both, although generally with increased potencies against 128 SA (Fig. 2a). 
a

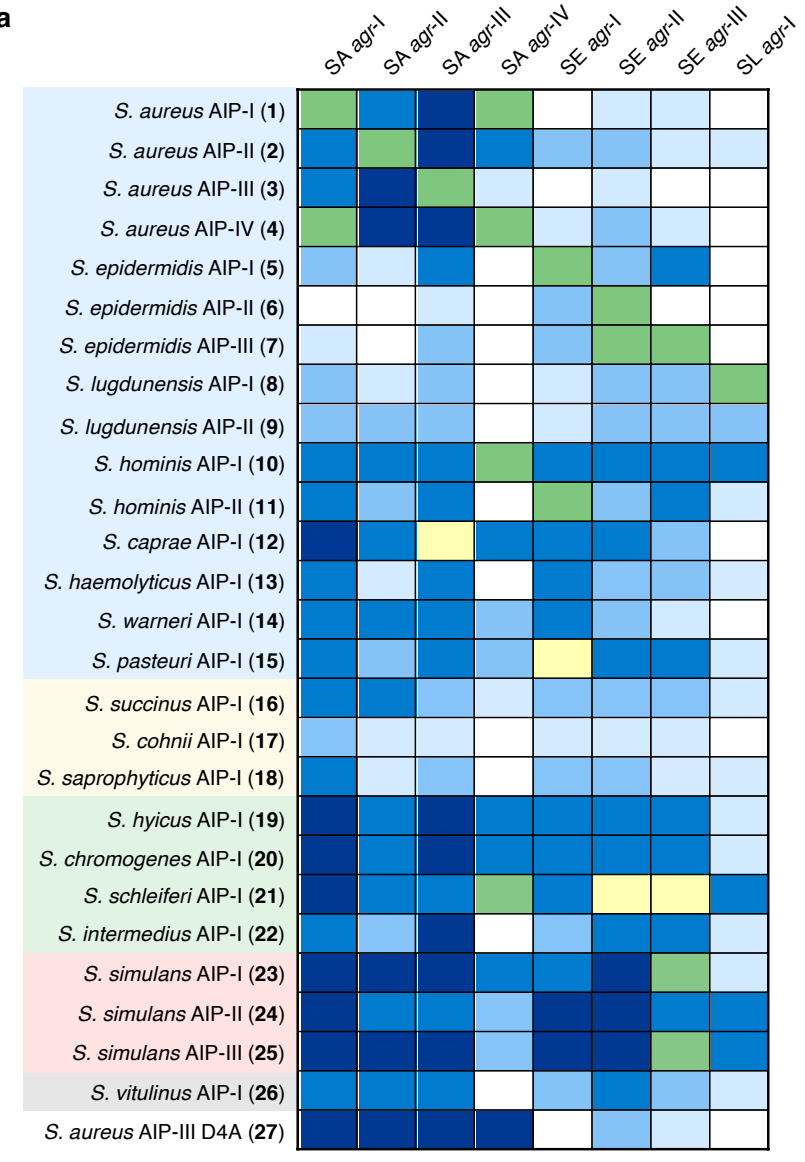

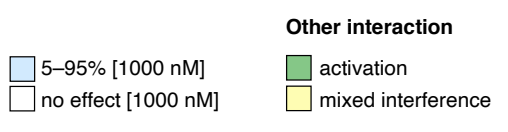

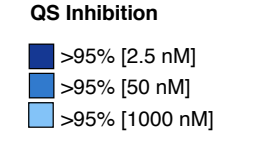

b

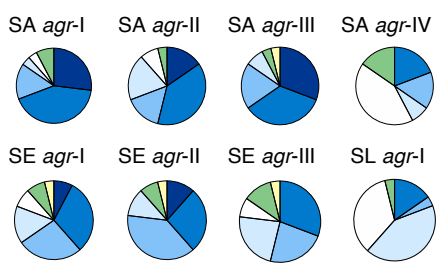

Total QS interactions

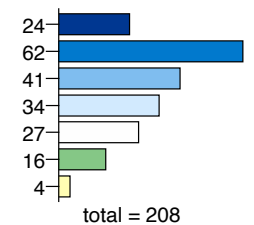

C
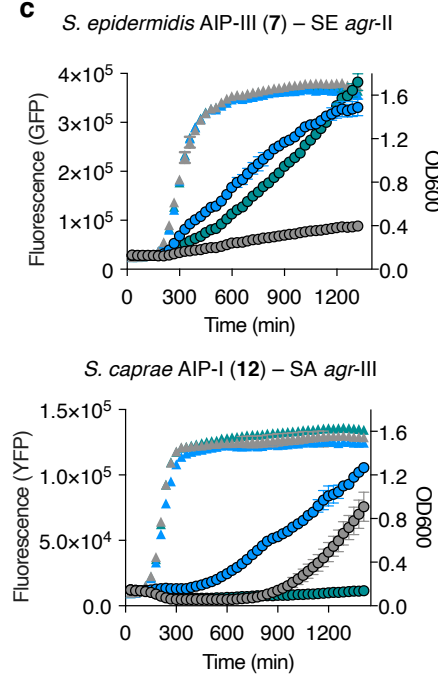

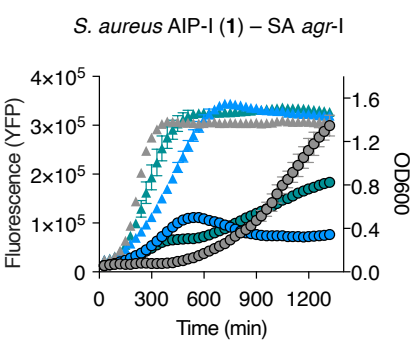

OFluorescence - agr activity

$\triangle$ OD600 - growth

no AlP added

AIP [1000 nM]

AIP $[50 \mathrm{nM}]$

Fig. 2 Quorum sensing (QS) interaction map. a, 27 synthetic AIPs were tested at several concentration $(1000 \mathrm{nM}, 50 \mathrm{nM}, 2.5 \mathrm{nM}$ ) against fluorescent reporter strains of S. aureus (SA) agr-I-IV, S. epidermidis (SE) agr-I-III and S. lugdunensis (SL) agr-I to assess their QS modulation abilities. Blue shading of boxes represents different potencies of QS inhibition ( $>95 \%$ at $2.5 \mathrm{nM}, 50 \mathrm{nM}$ and $1000 \mathrm{nM}$ or $5-95 \%$ at $1000 \mathrm{nM}$ ), white boxes represent no interaction at $1000 \mathrm{nM}$, green boxes represent QS activation and yellow boxes represent mixed interference (activation at $1000 \mathrm{nM}$ and inhibition at $50 \mathrm{nM}$ ). Shaded boxes represent the phylogenetic species groups as defined by Lamers et al. ${ }^{36}$ with following colors: Epidermidis-Aureus, blue; Saprophyticus, tan; Simulans, red; Hyicus-Intermedius, green; Sciuri, gray. All assays were performed as technical triplicates in three individual biological assays $(n=3)$. $\mathbf{b}$, Pie chart representing the summary of QS interaction for each species and agr group with the 26 native AIPs (excluding 27) as well as the overall summary of QS interactions following the heat map color scheme. c, Overnight growth $\left(\mathrm{OD}_{600}\right)$ and fluorescence curves recorded of bacterial cultures treated with selected AIPs at $1000 \mathrm{nM}$ or $50 \mathrm{nM}$ and without addition of AIPs. The graphs represent single experiments in technical duplicate.

The screening revealed that several AIPs increased the fluorescence readout compared to the control wells (Fig. 2a, shown in green) and we therefore monitored growth and fluorescence continuously overnight. Surprisingly, we found Se-AIP-III (7) to be an activator of SE agr-II, in contrast to previous experiments with bacterial supernatant where the AIP had no effect (Fig. 2c) ${ }^{35}$. Further, several crossspecies activators were discovered: S. hominis AIP-II (11) activated SE agr-I and S. simulans AIP-I 
(23) and AIP-III (25) activated SE agr-III (Supplementary Fig. 22). In addition, we monitored all

150 known activators in the same manner and observed earlier increase in fluorescence development,

151 which was often accompanied by a delay in growth as determined by $\mathrm{OD}_{600}$ values (Fig. $2 \mathrm{c}$ and

152 Supplementary Figs. 21 and 22). The previously inconsistent inhibition behavior of S. caprae AIP-I

153 (12) against SA agr-III (Extended Data Table 1, Supplementary Fig. 13) was also observed with the

154 fluorescent reporter strain, where activation occurred at $1 \mu \mathrm{M}$ while lower concentrations of the AIP

155 led to inhibition (Fig. 2c and Supplementary Fig. 17). The same mixed interference was seen for $S$.

156 pasteuri AIP-I (15) against SE agr-I (Supplementary Fig. 17) and for S. schleiferi AIP-I (21), which

157 showed 70\% inhibition of SE agr-II and III from 2.5-1000 nM. The non-natural SA pan-inhibitor 27

158 was a highly potent inhibitor of SA agr-I-IV, as expected, but showed low levels of inhibition against

159 SE $a g r-\mathrm{I}-\mathrm{III}$ and SL $a g r$-I. In contrast, the QS interaction map revealed pan-inhibitors of all 8

160 staphylococcal strains, namely S. hyicus AIP-I (19) and S. chromogenes AIP-I (20), which potently

161 inhibited all SA and SE agr variants but only weakly inhibited SL agr-I as well as S. simulans AIP-

162 II (24) that inhibited all 8 agr systems (Fig. 2a).

164 Quorum sensing interactions in the context of phylogeny of agr systems

165 The diversity of AIPs in the Staphylococcus genus can be estimated by the number of deposited AgrD

166 sequences in genomic databases. We collected the majority of available AgrD sequences and set as a 167 requirement for having a unique agr group that they possessed a unique AIP-containing sequence 168 (Extended Data Fig. 1b). A total of 125 AgrD sequences were found, belonging to 55 species, which 169 were used to construct a maximum likelihood tree (Fig. 4a). AgrD sequences with substitutions in the 170 C-terminal recognition sequences and the $N$-terminal leader peptide were not considered as a unique 171 agr group and were therefore not included. The agr groups of identified AIPs were named in their 172 chronological order of their discovery and agr groups with so far unknown AIPs were ranked in order 173 of their number of accessions in the Protein Database (Supplementary Table 3). The AgrD sequences 174 clustered to large extent into the 6 species groups that were previously defined by multi-locus analysis; 
albeit, with some exceptions ${ }^{36}$. Firstly, AgrD of SA agr-II is highly divergent from the other SA agr groups and is located on a single clade close to the Saprophyticus species group as found in a previous analysis of AgrD sequences ${ }^{39}$. The AgrD peptides of SL agr-I-II and S. caprae agr-I-II are phylogenetically divergent from the remaining Epidermidis-Aureus species group and show closer sequence similarity to the Hyicus-Intermedius and Simulans species groups, respectively. All AgrD sequences belonging to the Hyicus-Intermedius, the Simulans, and the Sciuri species groups clustered together and diverged early from the Epidermidis-Aureus and the Saprophyticus groups (Fig. 4a).

The QS interactions found in this group are relatively diverse, as it contains the three species against which the QS interaction screen was performed (Fig. 4b). The majority of AIPs that exhibit a lack of QS interference with SL agr-I belong to this species group.

The second largest species group, Saprophyticus, contains the smallest number of identified AIPs (3 exhibit relatively weak interactions with SA, SE and SL. The third largest species group, HyicusIntermedius, contains several species that are often isolated from animal hosts, such as $S$. hyicus, $S$. schleiferi, and $S$. chromogenes ${ }^{40}$. To date, 4 AIPs have been identified from the 25 unique agr groups, which are all potent interactors with the $a g r$ systems of SA, SE, and SL. The three known AIPs from the smaller Simulans species group (just 7 unique agr groups from 4 species) belong to $S$. simulans and show exceptionally strong QS interference with all 8 tested reporter strains.

Finally, the only discovered AIP of the Sciuri species group (7 agr groups from 5 species) exhibited moderate activity against most of the tested strains and none of the two agr groups in the Auricularis species group have had their AIPs identified yet. 
a

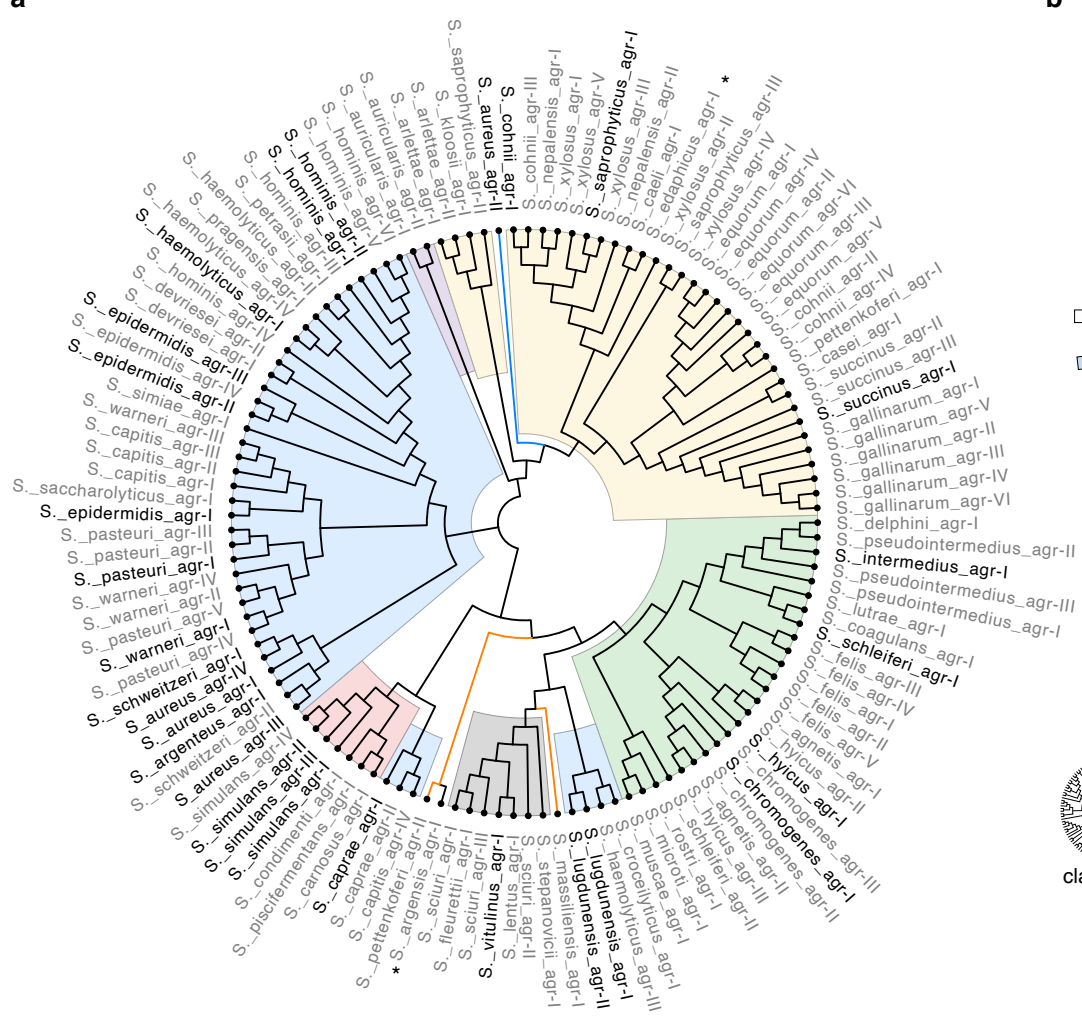

b

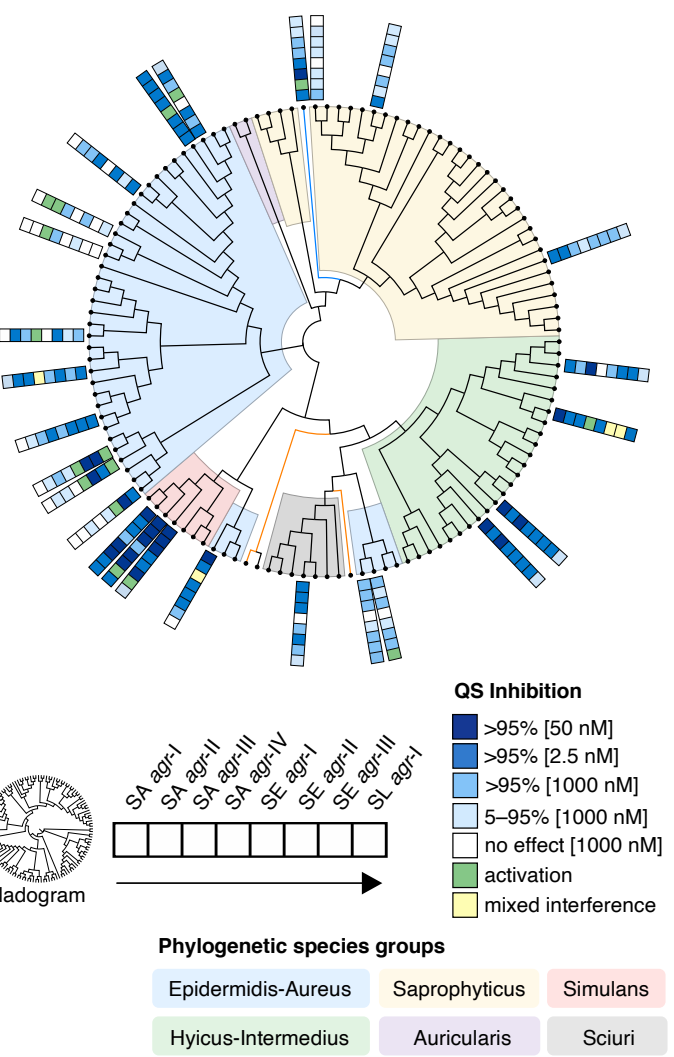

Fig. 3 | Phylogeny of the AIP-precursor peptides AgrD. a, Maximum likelihood tree (bootstrap $n=500$ ) created from 125 AgrD sequences with unique AIP-containing sequences. Names of agr groups with known AIPs are black and grey with unknown AIPs. Phylogenetic species groups as defined by Lamers et al. ${ }^{36}$ are colored as follows: Epidermidis-Aureus, blue; Saprophyticus, tan; Simulans, red; Hyicus-Intermedius, green; Auicularis, purple; Sciuri, gray. *Species not yet assigned to species group. b, Cladogram overlaid with QS interactions of known AIPs against SA $a g r-\mathrm{I}-\mathrm{IV}$, SE $a g r-\mathrm{I}-\mathrm{III}$ and SL $a g r$-I. The interactions of one AIP are represented as colored boxes with SA agr-I closest to the cladogram as depicted in the figure.

To further examine the diversity and relatedness of secreted AIPs, we created a maximum likelihood tree of the 12 amino acid-long AIP-containing sequences of all found AgrD peptides (Extended Data Fig. 4). Not unexpectedly, the clustering into species groups became less pronounced but several interesting observations surfaced. Notably, S. schleiferi agr-I and S. hominis agr-I, which both secrete activators of SA agr-IV, show high levels of relatedness and appear in close proximity in the cladogram. Also, high sequence similarity was revealed for SA agr-I, III, and IV to the Simulans species group, which diverge directly from the same clade. The most striking difference was the clustering of SE agr-II-IV into the Simulans species group. Particularly when considering that all interactions with full inhibition of SE at $2.5 \mathrm{nM}$ concentration of tested AIPs were elicited by $S$. 
simulans AIPs and the cross-species activation of SE agr-III was caused by two of the S. simulans

217 AIPs.

\section{The autoinducing peptides from $S$. simulans and chemical phylogenetics}

The most potent group of QS inhibitors revealed through our QS interaction mapping were the three AIPs produced by $S$. simulans (Figs. $2 \mathrm{a}$ and $4 \mathrm{a}$ ). We determined the $\mathrm{IC}_{50}$ values of $S$. simulans AIP-

I-III (23-25) by dose-response experiments against SA agr-I-IV, SE agr-I-III, and SL agr-I, which further confirmed their potent inhibitory properties (Extended Data Table 2 and Supplementary Figs.

(24), on the other hand, inhibited all 8 agr groups (SA, SE, SL); albeit, with slightly lower potencies against SA agr-II and SA agr-III compared to the other two S. simulans AIPs (Extended Data Table

2). The $S s$-AIP-I (23) and $S s$-AIP-III (25) have the same exotail (Fig. 4a), which is also shared by $S e$ AIP-III (7) and could therefore be the determining feature for the activation of SE agr-III. That $S s$ AIP-II (24) has a single amino acid substitution (N3Y) and acts as potent inhibitor of this system $\left(\mathrm{IC}_{50}=0.6 \mathrm{nM}\right)$ supports that hypothesis further. Similarly, it is tempting to speculate that the identical macrocycle sequence of $S s$-AIP-II (24) and Ss-AIP-III (25) may be important for the potent inhibition of SL agr-I by both AIPs. The potent inhibition of SE $a g r$-I-II by Ss-AIP-III (25) at subnanomolar $\mathrm{IC}_{50}$ values $(0.14 \mathrm{nM}$ and $0.11 \mathrm{nM})$ represent the most potent native QS interactions between previously reported inhibitors of this species group ${ }^{30}$.

238 We then performed a chemical phylogenetic analysis by combining the phylogenetic trees of the 8 239 AgrC receptors (SA, SE, SL) with determined $\mathrm{IC}_{50}$ values for the cognate AIPs of SA agr-I (1), SE agr-I (5), and SL agr-I (8) as well as pan-inhibitor Ss-AIP-II (24) (Fig. 4b, Extended Data Table 2 
242 distantly related to the other agr groups, creating a similar picture as observed in the phylogeny of

243 the full AgrD sequences (Fig. 3a).

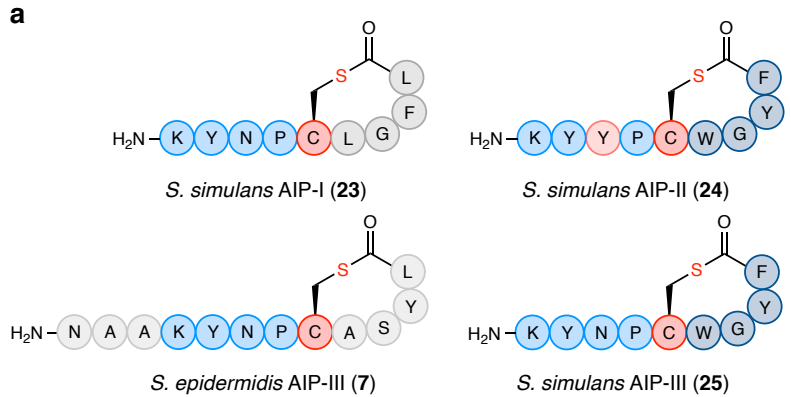

b
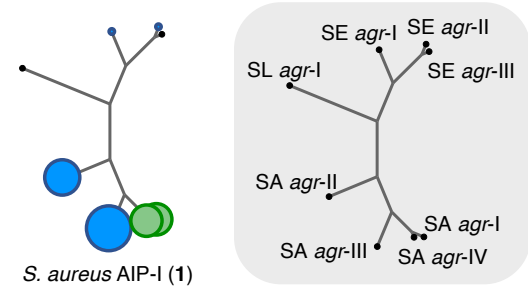

Inhibition $\mathrm{IC}_{50}(\mathrm{nM})$
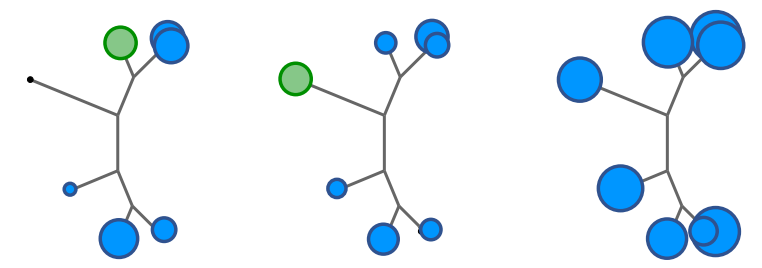

S. epidermidis AIP-I (5)

S. Iugdunensis AIP-I (8)

S. simulans AIP-II (24)

Fig. 4 | S. simulans AIP structures and chemical phylogenetic analyses. a, The structures of $S$. simulans AIP-I-III (23-25) and SE-AIP-III (7) show the same exotail amino acid sequence (KYNP) in 23, 25, and 7 as well as the same macrocyle amino acid sequence (CWGYF) for $\mathbf{2 4}$ and 25. b, Phylogenetic trees (bootstrap $n$ $=500$ ) of the AgrC receptor of SA $a g r-\mathrm{I}-\mathrm{IV}$, SE $a g r$-I-III, and SL agr-I were overlaid with $\mathrm{IC}_{50}$ values determined through full-dose response curves (Extended Data Table 2) highlighting the different QS interference patterns of cognate AIPs $(\mathbf{1}, \mathbf{5}$, and $\mathbf{8})$ and $S$. simulans AIP-II (24).

It is noteworthy that the receptors of $a g r$-I and $a g r$-IV share $87 \%$ sequence similarity despite their greatly differing susceptibly to QS interference (Fig. 2b) ${ }^{14}$. The AgrC receptors of SE also group together, with $a g r$-I being more distantly related to $a g r$-II/III, which parallels the AgrD clustering, while the AgrC receptor of SL is highly divergent from the other two species. The selective inhibition pattern of Sa-AIP-I (1) also observed in the QS interaction screening, appeared even more pronounced with nearly no interference with SE and SL (Fig. 4b). The Se-AIP-I (5) showed higher potency against SA agr-III than SE agr-II and III but no interference with SL agr-I. The cognate AIP 
bioRxiv preprint doi: https://doi.org/10.1101/2021.04.18.440348; this version posted April 19, 2021. The copyright holder for this preprint (which was not certified by peer review) is the author/funder, who has granted bioRxiv a license to display the preprint in perpetuity. It is made available under aCC-BY-NC-ND 4.0 International license.

of SL $a g r$-I (8) was active against all other agr groups, except SA $a g r$-IV. In contrast, Ss-AIP-II (24)

260 showed strong inhibitory properties in nearly all AIP-AgrC interactions regardless of the receptor 261 phylogeny.

\section{An autoinducing peptide from $S$. simulans attenuates MRSA in mouse skin model}

Next, we tested Ss-AIP-II (24) in an in vivo MRSA (agr-I) mouse skin infection model (Fig. 5a-d).

The importance of a functioning $a g r$ system for SA during infection to evade the immune response has been established and it was shown that inhibition of agr during early stages of infection can lead to improved disease outcome $48-72 \mathrm{~h}$ after its initiation ${ }^{41}$. Thus, Ss-AIP-II (24) $(100 \mu \mathrm{M})$ added to the MRSA inoculum $\left(10^{7} \mathrm{CFU}\right)$ applied on the skin was compared to vehicle and daily treatment with 269 the commercial antibacterial product Fucidin ${ }^{\circledR}$ (2\% fusidic acid). A significant reduction in the skin lesion size was observed after $48 \mathrm{~h}$ and $96 \mathrm{~h}$ for mice treated with $S s$-AIP-II (24) $(P=0.0174$ and $P$

$271=0.0249)$ as well as fusidic acid $(P=0.0108$ and $P=0.0202)$ compared to the vehicle control (Fig. 5a and c). Further, a significant decrease in bacterial load $(\sim 60$-fold, $P=0.009)$ was observed for 273 mice treated with Ss-AIP-II (24) compared to vehicle control after 4 days, which was comparable to 274 daily treatment with fusidic acid ( 38-fold, $P=0.0168)$ (Fig. 5b). No statistical difference between treatment or vehicle or time of the infection was found for the body weight, which was expected as no systemic infections were observed (Fig. 5d). These results are highly encouraging for the prospects of anti-virulence treatments of staphylococcal infections with non-antibiotic peptides. The higher extent of bacterial clearance is most likely a result of a more efficient immune response towards nonvirulent MRSA bacteria. 
a

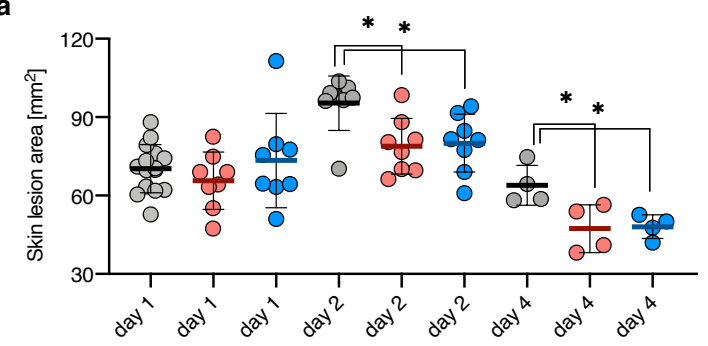

c

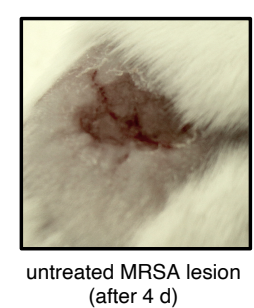

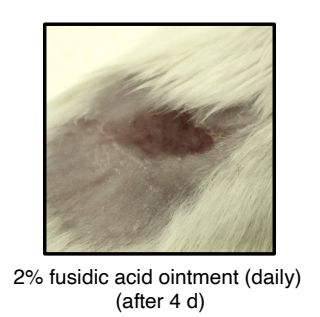

b
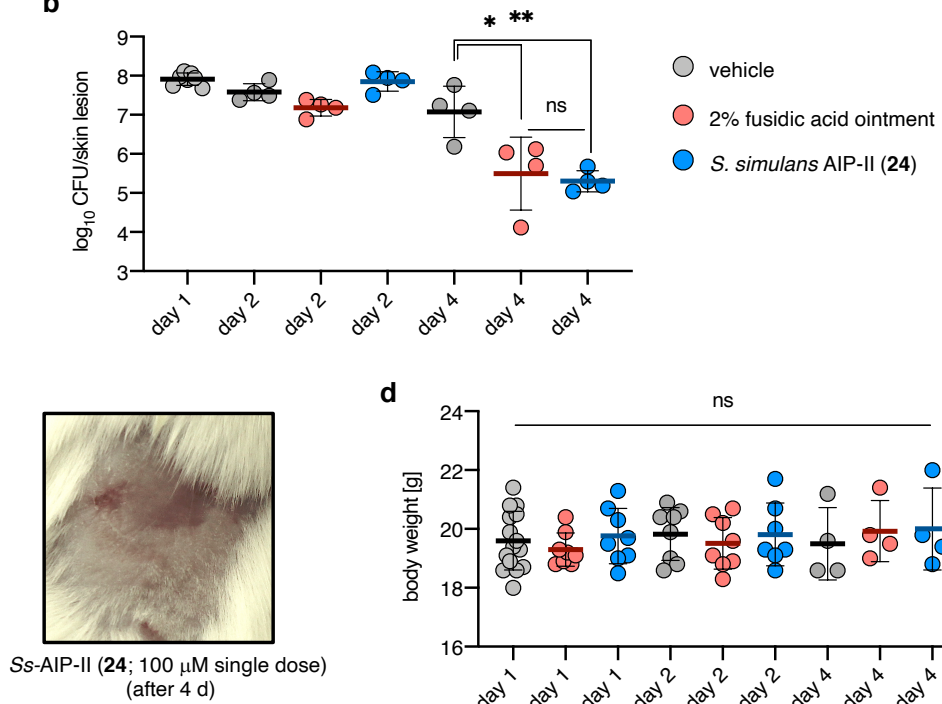

d

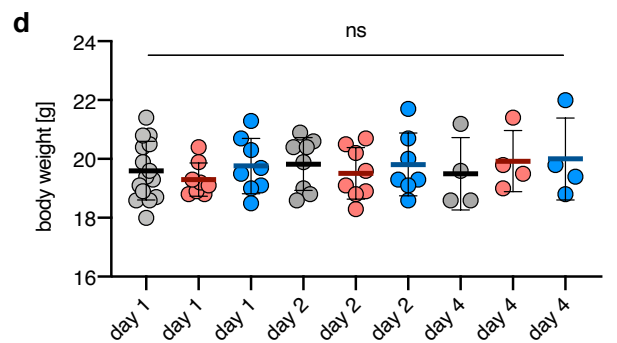

Fig. 5 | S. simulans AIP-II attenuates MRSA infection in murine skin model. a, Murine skin infection model performed with vehicle control group [day $1(n=16)$, day $2(n=8)$, day $4(n=4)$ ], fusidic acid (daily application of a $38.7 \mathrm{mM}$ ointment (Fucidin) [day $2(\mathrm{n}=8)$ and $4(\mathrm{n}=4)$ ] and 24 (single treatment at day 0 at $100 \mu \mathrm{M}$ [day $2(\mathrm{n}=8)$ and $4(\mathrm{n}=4)$ ]. MRSA inoculum: $10^{7} \mathrm{CFU}$. Skin lesions measured on day 1,2 , and 4 showing significant reduction in lesion size for mice treated with fusidic acid and 24 . $\mathbf{b}$, CFU count determined after day 1,2, and 4 showing significant reduction in bacterial load per skin lesion. c, Representative pictures taken of MRSA lesions at day 4 for untreated and treated mice. d, Body weight measured at day 1, 2, and 4 showing no statistically significant differences. Data is presented as mean and error bars represent the standard deviation (SD) of the mean. $P>0.05(\mathrm{~ns}), P<0.05(*), P<0.01(* *)$.

\section{Discussion}

Altering gene expression of competing staphylococci through secreted AIPs represents a powerful ability of the $a g r$ system next to its cognate function of "sensing" cell density. Even though agr loci and AgrD peptides can be found in the genomes of all species it is not known if and to what extent these systems are functional. However, 21 AIPs from 15 CoNS have now been identified from bacterial supernatants pointing towards a broad utilization of the agr system, including secretion and detection of AIPs as well as potential QS interference. The colonization of humans and animals by a wide range of staphylococcal species emphasizes that many species share the same environments, 
providing an arena for biologically relevant inter-staphylococcal interactions, such as QS interference.

300 We report herein an improvement of our previously developed method for AIP identification, which 301 allows direct trapping of AIPs in untreated supernatants, which will further help to elucidate the complex network of QS interactions. The faster analysis rate enabled us to rapidly screen $46 \mathrm{SE}$ samples from human nasal swabs with an identification frequency of more than $70 \%$ of the samples. In addition, the method was used to identify 3 new AIPs, $S$. pasteuri AIP-I (15), S. succinus AIP-I (16), and S. cohnii AIP-I (17) that helped improve coverage of the various species groups. Combined with the previously identified AIPs, we determined QS modulating properties against fluorescent reporter strains of SA $a g r-\mathrm{I}-\mathrm{IV}, \mathrm{SE}$ agr-I-III and SL $a g r$-I to provide a map of $>200$ QS interactions. This represents the largest systematic analysis of the currently known staphylococcal QS landscape and reveals extensive promiscuity of staphylococcal crosstalk, leading to the discovery of several 310 cross-species activators as well as pan-inhibitors against all the 8 tested $a g r$ systems. To further 311 elucidate the diversity of AIPs from the Staphylococcus genus and the phylogenetic relatedness 312 between agr groups, we collected 125 AgrD sequences of 55 species and created cladograms that 313 together with the QS interaction data showed intriguing correlations between sequence similarity and 314 bioactivity. For example, the interactions between the AIPs of S. simulans and the agr system of SE 315 were highly sequence sensitive with a single substitution deciding between potent inhibitor and 316 activator of SE agr-III. We could further show a significant effect of an inhibitory AIP, produced by 317 S. simulans (24), on the colonization and pathogenesis of MRSA, highlighting the power of gene 318 repression through QS inhibition ${ }^{21,23,24,28}$. In addition, the interactions of S. simulans AIPs with SL $319 a g r$-I represents the first highly potent inhibitors of QS in SL with a 400-fold increase in potency 320 compared to the previously reported compounds.

321 The impact of the substantial native QS interference among commensal staphylococci in human 322 microbiota may reflect the considerable overlap in living space but this remains to be explored further. 323 However, our results highlight the potential importance of the agr system and cross-species 
324 interference on the colonization of commensal staphylococci and on the pathogenesis of for example 325 SA.

326 It is our hope that the mapping of cross-species QS interactions initiated in the present work will help

327 provide insight into the roles of agr systems in future investigations. Furthermore, our findings

328 highlight the potential utility of natural scaffolds as a promising platform for the development of 329 inhibitors for anti-virulence treatment of Staphylococcus infections.

$331 \quad$ Methods

332 The chemical synthesis details, characterization data and copies of NMR spectra are provided in the 333 Supplementary Information.

\section{Reagents and materials}

336 Bacteria were cultured in tryptic soya broth (TSB) medium and on tryptic soya agar (TSA) plates 337 (both Oxoid) supplemented with $10 \mu \mathrm{g} / \mathrm{ml}$ chloramphenicol (CAM) or erythromycin (ERM) (Sigma338 Aldrich) when appropriate. $N \alpha$-Fmoc protected and $\alpha-N$-Boc protected amino acids, 2-(1H339 Benzotriazol-1-yl)-1,1,3,3-tetramethyluronoium hexafluorophosphate (HBTU) and 2-(1H-7340 Azabenzotriazol-1-yl)-1,1,3,3-tetramethyluronoium hexafluorphosphate (HATU) were obtained 341 from ChemImpex and PepChem. Aminomethyl ChemMatrix resin was obtained from PCAS 342 BioMatrix. Aminomethyl PEGA resin, N,N-diisopropylethylamine, 4-nitrophenylchloroformate, 343 maleic acid (qNMR grade) and nitrocefin were obtained from Sigma-Aldrich. All other chemicals 344 used were obtained in the highest available purity from CombiBlocks or IrisBiotech. All solvents 345 used were of analytical grade and purchased from Fisher Scientific. Manual solid-phase peptide 346 synthesis was performed in polypropylene syringes equipped with fritted disks, purchased from 347 Torviq. 


\section{Compound analysis and purification}

351 Analytical ultra-performance liquid chromatography (UPLC) analyses were performed on a C18 352 Agilent InfinityLab Poroshell 120 column $(2.7 \mu \mathrm{m}, 100 \times 3.0 \mathrm{~mm})$ using an Agilent 1260 Infinity II series system equipped with a diode array UV detector. A gradient with eluent A (water-MeCNTFA, 95:5:0.1, v/v/v) and eluent $\mathrm{B}(0.1 \% \mathrm{TFA}$ in $\mathrm{MeCN})$ rising linearly from 0 to $50 \%$ of $\mathrm{B}$ over $10.0 \mathrm{~min}$ at a flow rate of $1.2 \mathrm{~mL} \mathrm{~min}^{-1}$ was applied to determine the purity of peptides $(\lambda=215 \mathrm{~nm})$. UPLC-mass spectrometry (MS) analyses were performed on a Phenomenex Kinetex column (1.7 $\mu \mathrm{m}$, $100 \AA$, $50 \times 2.10 \mathrm{~mm})$ using a Waters Acquity system. A gradient with eluent $\mathrm{C}(0.1 \% \mathrm{HCOOH}$ in water $)$ and eluent $\mathrm{D}(0.1 \% \mathrm{HCOOH}$ in $\mathrm{MeCN})$ rising linearly from 0 to $95 \%$ of $\mathrm{D}$ over $5.20 \mathrm{~min}$ at a flow rate of $0.6 \mathrm{~mL} \mathrm{~min}^{-1}$ was applied to analyse reaction mixtures. Preparative high-performance

360 liquid chromatography (HPLC) purification was performed on a C18 Phenomenex Luna column $361(5 \mu \mathrm{m}, 100 \AA, 250 \times 20 \mathrm{~mm})$ using an Agilent $1260 \mathrm{LC}$ system equipped with a diode array 362 ultraviolet detector. Various gradients with eluent A and eluent B at a flow rate of $20 \mathrm{~mL} \mathrm{~min}^{-1}$ were applied for the purification. Fractions containing the purified target peptide were identified using UPLC-MS or matrix assisted laser desorption ionisation-time of flight mass spectrometry (MALDI365 TOF MS). MALDI-TOF mass spectra were recorded with a Bruker microflex bench-top MALDI using a matrix of alpha-cyano-4-hydroxycinnamic acid or 2,5-dihydroxybenzoic acid in water/MeCN $(1: 1, \mathrm{v} / \mathrm{v})$ containing $0.1 \%$ TFA. The observed $\mathrm{m} / \mathrm{z}$ corresponded to the monoisotopic ions, unless otherwise stated. Nuclear magnetic resonance (NMR) spectra were recorded at $298 \mathrm{~K}$ using a Bruker 369 Avance III HD ( ${ }^{1} \mathrm{H}$ NMR and ${ }^{13} \mathrm{C}$ NMR recorded at $600 \mathrm{MHz}$ and $150 \mathrm{MHz}$, respectively). Chemical 370 shifts are reported in parts per million (ppm) relative to the deuterated solvent peak of DMSO- $d_{6}\left(\delta_{\mathrm{H}}\right.$ $\left.371=2.50 \mathrm{ppm} ; \delta_{\mathrm{C}}=39.52 \mathrm{ppm}\right)$ or $\mathrm{CDCl}_{3}\left(\delta_{\mathrm{H}}=7.26 \mathrm{ppm} ; \delta_{\mathrm{C}}=77.16 \mathrm{ppm}\right)$ as internal standard. 


\section{NCL trapping of AIPs from bacterial supernatants}

Bacterial isolates were streaked on agar plates and grown over night at $37^{\circ} \mathrm{C}$. Single colonies were then inoculated in $50 \mathrm{~mL}$ TSB media overnight at $37^{\circ} \mathrm{C}$ in an incubator at $200 \mathrm{rpm}$ shaking. Overnight cultures were centrifuged at $8000 \mathrm{rpm}$ at $4{ }^{\circ} \mathrm{C}$ and supernatants filtered through a sterile filter $(0.22 \mu \mathrm{m})$ and stored at $4{ }^{\circ} \mathrm{C}$ for direct use or frozen and stored at $-20{ }^{\circ} \mathrm{C}$ until use. Resin preparation: Fmoc-Cys(St-Bu)-Rink-PEGA resin (50 mg) or Fmoc-Cys(STmp)-Rink-PEGA resin (50 mg) was placed in a $2.0 \mathrm{~mL}$ polypropylene syringe equipped with a fritted disk, swelled in DMF for $15 \mathrm{~min}$ and washed with DMF $(5 \times 1 \mathrm{~min})$. The resin was treated with piperidine in DMF $(1: 4$, $\mathrm{v} / \mathrm{v}, 2.0 \mathrm{~mL})(1 \times 2 \mathrm{~min}, 1 \times 20 \mathrm{~min})$ and washed with $\mathrm{DMF}(5 \times 1 \mathrm{~min})$. The resin was then treated with a solution of $\beta$-mercatoethanol (BME) in $\operatorname{DMF}(1: 4, \mathrm{v} / \mathrm{v}, 2.0 \mathrm{~mL})$ containing $N$-methyl morpholine (NMM) (0.1 M) or DL-dithiothreitol (DTT) in DMF (0.05:0.95, w/v, $2.0 \mathrm{~mL})$ containing $\operatorname{NMM}(0.1 \mathrm{M})(3 \times 10 \mathrm{~min})$ and subsequently washed with DMF $(3 \times 1 \mathrm{~min}), \mathrm{MeOH}(3 \times 1 \mathrm{~min})$ and $\mathrm{H}_{2} \mathrm{O}(3 \times 1 \mathrm{~min})$. NCL trapping: The sterile and filtered bacterial supernatant $(\sim 50 \mathrm{~mL})$ was added to $50 \mathrm{~mL}$ centrifugal tube and the $\mathrm{pH}$ adjusted to $\mathrm{pH}=\sim 7.0$ using aqueous $\mathrm{NaOH}(1.0 \mathrm{M})$. An aqueous tris(2-carboxyethyl)phosphine hydrochloride (TCEP) solution (1.0 mL, 0.5 M, pH = 7.0; final conc. $=10.0 \mathrm{mM}$ ) was added to the supernatant followed by the deprotected Cys-Rink-PEGA-resin and the centrifugal tube containing the trapping mixture was agitated at $37^{\circ} \mathrm{C}$ overnight. The next day, the resin was separated from the supernatant through filtration using a $10 \mathrm{~mL}$ polypropylene syringe equipped with a fritted disk under suction and washed with DMF $(3 \times 1 \mathrm{~min}), \mathrm{H}_{2} \mathrm{O}(3 \times 1 \mathrm{~min})$, and DMF $(3 \times 1 \mathrm{~min})$. A solution of DTT in DMF $(0.05: 0.95, \mathrm{w} / \mathrm{v}, 2.0 \mathrm{~mL})$ containing NMM $(0.1 \mathrm{M})$ was added to the resin and the resin was agitated at $37^{\circ} \mathrm{C}$. After $30 \mathrm{~min}$, the resin was washed with DMF $(3 \times 1 \mathrm{~min}), \mathrm{MeOH}(3 \times 1 \mathrm{~min})$, and $\mathrm{CH}_{2} \mathrm{Cl}_{2}(3 \times 1 \mathrm{~min})$ and dried under suction for $15 \mathrm{~min}$. The dried resin was treated with a cleavage cocktail $(2.0 \mathrm{~mL}$, TFA-MilliQ water, 97:3, v/v) for $2 \mathrm{~h}$ at room temperature. The peptide containing cleavage solution was removed from the resin, collected and the resin rinsed with neat TFA $(1.0 \mathrm{~mL})$. The combined TFA fractions were evaporated under $\mathrm{N}_{2}$ 
stream to near dryness, redissolved in a solution of $\mathrm{MeCN}$ in $\mathrm{H}_{2} \mathrm{O}(100 \mu \mathrm{L}, 1: 1, \mathrm{v} / \mathrm{v})$ and filtered $(0.22 \mu \mathrm{m})$. LC-MS analysis: The filtered TFA cleavage solution was analysed using an Waters Acquity system equipped with a Phenomenex Kinetex column $(1.7 \mu \mathrm{m}, 100 \AA, 50 \times 2.10 \mathrm{~mm})$ applying a gradient with eluent $\mathrm{C}(0.1 \% \mathrm{HCOOH}$ in water) and eluent $\mathrm{D}(0.1 \% \mathrm{HCOOH}$ in $\mathrm{MeCN})$ rising linearly from 0 to $50 \%$ of $\mathrm{D}$ over $10.0 \mathrm{~min}$ at a flow rate of $0.6 \mathrm{~mL} \mathrm{\operatorname {min } ^ { 1 }}$ and an injection volume of $40 \mu \mathrm{L}$. The total ion chromatograms (TIC) were analyzed by displaying extracted ion chromatograms (EIC) of $\mathrm{m} / z[\mathrm{M}+\mathrm{H}]^{+}$values of the possible linear peptides with an additional $C$ terminal cysteine and amide functionality based on the AgrD sequence.

\section{Concentration determination of DMSO stock solutions}

Concentrations of DMSO stock solutions for compounds were determined by quantitative NMR relative to the signal $\left(\delta_{\mathrm{H}}=6.24 \mathrm{ppm}, 2 \mathrm{H}\right)$ of the internal standard maleic acid (qNMR grade).

\section{$\beta$-Lactamase assay for $\mathrm{IC}_{50}$ determination against $S$. aureus agr-I-IV}

The $\beta$-lactamase reporter strains RN10829 [(P2-agrA:P3-blaZ) $]^{42}$, with pagrC-I ${ }^{43}$, pagrC-II, pagrCIII or pagrC-IV ${ }^{34}$ substituting the native $a g r$ locus with a chromosomal integration of P2-agrA and P3-blaZ and a plasmid from which a wild-type variant of the corresponding AgrC is expressed, were used to asses inhibition and activation of the AgrC receptor via $\beta$-lactamase activity in response to varying concentrations of the QS modulating peptides. Overnight cultures of the reporter strains in TSB medium were diluted 1:250 in fresh TSB medium and grown to $\mathrm{OD}_{600}=0.35-0.40$ (early exponential phase) at $37^{\circ} \mathrm{C}$. Peptide solutions $(10 \mu \mathrm{L})$ in 1:10 serial dilutions from DMSO stock solutions $(1 \mathrm{mM})$ in TSB medium (final concentrations $=10 \mu \mathrm{M}-10 \mathrm{pM}$ ) were added to each well of a clear 96-well plate as well as solutions $(10 \mu \mathrm{L})$ of cognate AIP (final concentration $=100 \mathrm{nM})$ in TSB medium followed by $80 \mu \mathrm{L}$ of bacterial cells. Control wells for $100 \% \beta$-lactamase activity were wells replacing peptide solution with TSB media $(10 \mu \mathrm{L})$ and control wells for $0 \% \beta$-lactamase 
activity were wells replacing both peptide and cognate AIP solutions with TSB medium $(20 \mu \mathrm{L})$. The

$80{ }^{\circ} \mathrm{C}$ to minimize growth during nitrocefin treatment. Next, the $96-w e l l$ plates were thawed and

$\mathrm{OD}_{600}$ values were determined using a plate-reader followed by addition of $50 \mu \mathrm{L}$ of nitrocefin

solution to the wells (final concentration $=33.3 \mu \mathrm{g} / \mathrm{mL}$ ). $\beta$-Lactamase activity was monitored at

$\mathrm{OD}_{486}$ every $20 \mathrm{~s}$ for $10 \mathrm{~min}$ at $37^{\circ} \mathrm{C}$ using a plate-reader. Linear nitrocefin conversion rates were

431 plotted to obtain $\mathrm{IC}_{50}$ values by non-linear regression with variable slope using GraphPad Prism 8.0 software. Assays were performed at least as duplicate determinations in biological triplicate.

\section{Fluorescence reporter assay for screening agr-interference of AIPs}

435 Peptides were evaluated for the ability to interfere with agr-mediated quorum sensing in S. aureus 436 (AH1677, AH430, AH1747 and AH1872 for agr-I-IV, respectively) ${ }^{44}$ using reporter strains expressing yellow fluorescent protein (YFP) upon agr activation. Interference with agr-mediated quorum sensing in S. epidermidis (AH3408, AH3623 and AH3409 for agr-I-III, respectively) ${ }^{35}$ and

S. lugdunensis (AH4031 for agr-I) ${ }^{30}$ was evaluated using reporter strains expressing superfolder green fluorescent protein (sGFP) upon agr activation. Overnight cultures of the reporter strains were grown in TSB medium containing chloramphenicol (CAM, $10 \mu \mathrm{g} / \mathrm{mL}$ for $S$. aureus) or erythromycin containing the same antibiotic. Assays were performed in sterile black 96-well plates with clear

444 bottom and the outer wells were filled with water $(200 \mu \mathrm{L})$ to ensure consistent bacterial growth in the remaining 60 wells. All peptides were screened at concentrations of $1.0 \mu \mathrm{M}$ and $50 \mathrm{nM}$ and peptides showing at least $75 \%$ inhibition at $50 \mathrm{nM}$ were screened further at $2.5 \mathrm{nM}$ against the 447 respective reporter strain, and similarly at $0.125 \mathrm{nM}$ in the case of at least $75 \%$ inhibition at $2.5 \mathrm{nM}$. 448 DMSO stock solutions of peptides $(1 \mathrm{mM})$ were diluted in TSB media and added $(15 \mu \mathrm{L})$ in technical 449 triplicate to the 96-well plate followed by diluted bacterial overnight cultures $(135 \mu \mathrm{L})$. Control wells 
450 for $100 \%$ agr acitivty were wells replacing the peptide solution with TSB medium $(15 \mu \mathrm{L})$. Wells 451 containing $150 \mu \mathrm{L}$ TSB medium were used to measure background fluorescence. The 96-well plates 452 were incubated on a shaking table at $400 \mathrm{rpm}$ at $37^{\circ} \mathrm{C}$ for $22-24 \mathrm{~h}$ and fluorescence (for GFP: 453 extication $479 \mathrm{~nm}$, emission $520 \mathrm{~nm}$; for YFP: extication $500 \mathrm{~nm}$, emission $541 \mathrm{~nm}$; automatic gain) 454 and $\mathrm{OD}_{600}$ values were subsequently measured using a plate-reader. Background fluorescence was 455 subtracted from all wells and further normalized to the corresponding $\mathrm{OD}_{600}$ value of the respective 456 wells. Average fluorescence of control wells was used as relative measure for $100 \%$ activation of the agr-circuit and bar graphs were generated using GraphPad Prism 8.0 software. All assays were performed in biological triplicate.

\section{Fluorescence reporter assay for $\mathrm{IC}_{50}$ determination}

461 Overnight cultures of the reporter strains were grown in TSB medium containing chloramphenicol 462 (CAM, $10 \mu \mathrm{g} / \mathrm{mL}$ for $S$. aureus) or erythromycin (ERM, $10 \mu \mathrm{g} / \mathrm{mL}$ of $S$. epidermidis and $S$. 463 lugdunensis) and diluted 1:100 in fresh TSB medium containing the same anitbiotic. Assays were 464 performed in sterile black 96-well plates with clear bottom. Peptide solutions $(15 \mu \mathrm{L})$ in 1:5 serial 465 dilutions from DMSO stock solutions $(1 \mathrm{mM})$ in TSB medium were added to the 96-well plate in 466 technical duplicate followed diluted bacterial overnight cultures $(135 \mu \mathrm{L})$. Control wells for $100 \%$ 467 agr acitivty were wells replacing the peptide solution with TSB medium $(15 \mu \mathrm{L})$. Wells containing $468150 \mu \mathrm{L}$ TSB medium were used to measure background fluorescence. The 96-well plates were 469 incubated in a humidified incubator at $37^{\circ} \mathrm{C}$ shaking at $1000 \mathrm{rpm}$ for $22-24 \mathrm{~h}$ and fluorescence (for 470 GFP: extication $479 \mathrm{~nm}$, emission $520 \mathrm{~nm}$; for YFP: extication $500 \mathrm{~nm}$, emission $541 \mathrm{~nm}$; automatic 471 gain) and $\mathrm{OD}_{600}$ values were subsequently measured using a plate-reader. Background fluorescence 472 was subtracted from all wells and further normalized to the corresponding $\mathrm{OD}_{600}$ value of the 473 respective wells. Average fluorescence of control wells were used as relative measure for $100 \%$ 474 activation of the agr-circuit. Relative $a g r$ activity was plotted to obtain $\mathrm{IC}_{50}$ values by non-linear 
regression with variable slope using GraphPad Prism 8.4 software. All assays were performed in biological triplicate.

\section{Maximum-likelihood tree construction for AgrD sequences}

AgrD sequences (125) were retrieved from the protein database of the National Center of

Biotechnology Information (NCBI). Multiple sequence alignment was perfomed using the program

481 ClustalW $^{45}$ in MEGA X (10.1.18) and maximum-likelihood trees were constructed through 500 bootstrap replicates using the Jones-Taylor-Thornton (JTT) model ${ }^{46}$ in MEGA X (10.1.18).

\section{In vivo MRSA skin infection model}

The mouse model was performed under contract at Statens Serum Institut (DK) essentially as previously described ${ }^{47}$. In brief, eight to ten-week-old Balb/c female mice (Taconic Denmark) mice were used for all experiments $\left[n=16\right.$ for vehicle, $n=8$ for Fucidin ${ }^{\circledR}$ ( $2 \%$ fusidic acid ointment) removed on a $2 \mathrm{~cm}^{2}$ skin area on the back and thereafter was the outer most layer of the skin scraped off with a dermal curette to obtain a $1 \mathrm{~cm}^{2}$ superficial skin lesion. For vehicle control and Fucidin ${ }^{\circledR}$ treatment, $10 \mu \mathrm{L}$ inoculum containing approximatly $10^{7} \mathrm{CFU}$ of methilicin-resistant $S$. aureus 
on day 1,2 , and 4 of all mice. Mice were sacrificed on day $1(n=8$ vehicle), day $2(n=4$ vehilce,

501 Fucidin $\left.^{\circledR}, 24\right)$ and day $4\left(\mathrm{n}=4\right.$ vehilce, Fucidin $\left.{ }^{\circledR}, 24\right)$ and the infected skin area was cut out and

502 homogenized to determine the CFU count in the skin lesions.

\section{Statistical analysis}

505 All statistical analyses were performed using GraphPad Prism 8.4 software. $P$ values were determined using one-way analysis of variance (ANOVA) and Dunnet's test. $P$ values $<0.05$ considered significant.

\section{References}

5101 Euzéby, J. P. List of Bacterial Names with Standing in Nomenclature: a Folder Available on 511 the Internet. Int. J. Syst. Evol. Microbiol. 47, 590-592 (1997). (Website accessed March 2021)

5122 Otto, M. Staphylococci in the human microbiome: the role of host and interbacterial 513 interactions. Curr. Opin. Microbiol. 53, 71-77 (2020).

5143 Parlet, C. P., Brown, M. M. \& Horswill, A. R. Commensal Staphylococci Influence Staphylococcus aureus Skin Colonization and Disease. Trends Microbiol. 27, 497-507 (2019).

5164 David, M. Z. \& Daum, R. S. Community-associated methicillin-resistant Staphylococcus aureus: epidemiology and clinical consequences of an emerging epidemic. Clin. Microbiol. Rev. 23, 616-687 (2010).

5195 DeLeo, F. R., Otto, M., Kreiswirth, B. N. \& Chambers, H. F. Community-associated meticillin-resistant Staphylococcus aureus. Lancet 375, 1557-1568 (2010).

5216 Lee, A. S. et al. Methicillin-resistant Staphylococcus aureus. Nat. Rev. Dis. Primers 4, 18033 (2018).

Becker, K., Heilmann, C. \& Peters, G. Coagulase-Negative Staphylococci. Clin. Microbiol. Rev. 27, 870-926 (2014).

5258 Rogers, K. L., Fey, P. D. \& Rupp, M. E. Coagulase-Negative Staphylococcal Infections. Infect. 526 Dis. Clin. North Am. 23, 73-98 (2009).

5279 Otto, M. Staphylococcus epidermidis - the 'accidental' pathogen. Nat. Rev. Microbiol. 7, 555-567 (2009). 
Brown, M. M. \& Horswill, A. R. Staphylococcus epidermidis-Skin friend or foe? PLoS Pathog. 16, e1009026 (2020).

Liu, Q. et al. Staphylococcus epidermidis Contributes to Healthy Maturation of the Nasal Microbiome by Stimulating Antimicrobial Peptide Production. Cell Host Microbe 27, 68-78 (2020).

Linehan, J. L. et al. Non-classical Immunity Controls Microbiota Impact on Skin Immunity and Tissue Repair. Cell 172, 784-796 (2018).

Byrd, A. L., Belkaid, Y. \& Segre, J. A. The human skin microbiome. Nat. Rev. Microbiol. 16, 143-155 (2018).

Thoendel, M., Kavanaugh, J. S., Flack, C. E. \& Horswill, A. R. Peptide signaling in the Staphylococci. Chem. Rev. 111, 117-151 (2011).

Wang, B. \& Muir, T. W. Regulation of virulence in Staphylococcus aureus: molecular mechanisms and remaining puzzles. Cell Chem. Biol. 23, 214-224 (2016).

Ji, G., Beavis, R. C. \& Novick, R. P. Cell density control of staphylococcal virulence mediated by an octapeptide pheromone. Proc. Natl. Acad. Sci. U. S. A. 92, 12055-12059 (1995). Variants. Science 276, 2027-2030 (1997).

Dickey, S. W., Cheung, G. Y. C. \& Otto, M. Different drugs for bad bugs: antivirulence strategies in the age of antibiotic resistance. Nat. Rev. Drug. Discov. 16, 457-471 (2017).

Canovas, J. et al. Cross-talk between Staphylococcus aureus and other staphylococcal species via the agr quorum sensing system. Front. Microbiol. 7, 1733 (2016).

Gless, B. H. et al. Structure-activity relationship study based on autoinducing peptide (AIP) from dog pathogen S. schleiferi. Org. Lett. 19, 5276-5279 (2017).

Paharik, A. E. et al. Coagulase-negative staphylococcal strain prevents Staphylococcus aureus colonization and skin infection by blocking quorum sensing. Cell Host Microbe 22, 111 (2017).

22 Peng, P. et al. Effect of Co-inhabiting Coagulase Negative Staphylococci on S. aureus agr Quorum Sensing, Host Factor Binding, and Biofilm Formation. Front. Microbiol. 10, 2212 (2019).

23 Williams, M. R. et al. Quorum sensing between bacterial species on the skin protects against epidermal injury in atopic dermatitis. Sci. Transl. Med. 11, eaat8329 (2019). Brown, M. M. et al. Novel Peptide from Commensal Staphylococcus simulans Blocks Methicillin-Resistant Staphylococcus aureus Quorum Sensing and Protects Host Skin from Damage. Antimicrob. Agents Chemother. 64, e00172-00120 (2020). 

in the agr autoinducing peptides of Staphylococcus aureus. Biochemistry 41, 10095-10104 (2002). inhibitors of quorum sensing in Staphylococcus aureus revealed through a systematic synthetic study of the group-III autoinducing peptide. J. Am. Chem. Soc. 135, 7869-7882 (2013).

Vasquez, J. K. \& Blackwell, H. E. Simplified Autoinducing Peptide Mimetics with SingleNanomolar Activity Against the Staphylococcus aureus AgrC Quorum Sensing Receptor. ACS Infect. Dis. 5, 484-492 (2019).

Nakatsuji, T. et al. Development of a human skin commensal microbe for bacteriotherapy of atopic dermatitis and use in a phase 1 randomized clinical trial. Nat. Med. (2021). DOI: 10.1038/s41591-021-01256-2

Heilbronner, S. \& Foster, T. J. Staphylococcus lugdunensis: a Skin Commensal with Invasive Pathogenic Potential. Clin. Microbiol. Rev. 34, e00205-00220 (2021).

Gordon, C. P., Olson, S. D., Lister, J. L., Kavanaugh, J. S. \& Horswill, A. R. Truncated Autoinducing Peptides as Antagonists of Staphylococcus lugdunensis Quorum Sensing. J. Med. Chem. 59, 8879-8888 (2016).

Otto, M., Echner, H., Voelter, W. \& Götz, F. Pheromone Cross-Inhibition between Staphylococcus aureus and Staphylococcus epidermidis. Infect. Immun. 69, 1957-1960 (2001). Yang, T., Tal-Gan, Y., Paharik, A. E., Horswill, A. R. \& Blackwell, H. E. Structure-function analyses of a Staphylococcus epidermidis autoinducing peptide reveals motifs critical for AgrC-type receptor modulation. ACS Chem. Biol. 11, 1982-1991 (2016).

Vasquez, J. K. et al. Conformational Switch to a $\beta$-Turn in a Staphylococcal Quorum Sensing Signal Peptide Causes a Dramatic Increase in Potency. J. Am. Chem. Soc. 142, 750-761 (2020). Gless, B. H. et al. Identification of autoinducing thiodepsipeptides from staphylococci enabled by native chemical ligation. Nat. Chem. 11, 463-469 (2019).

Olson, M. E. et al. Staphylococcus epidermidis agr quorum-sensing system: signal identification, cross talk, and importance in colonization. J. Bacteriol. 196, 3482-3493 (2014).

\footnotetext{
Lamers, R. P. et al. Phylogenetic relationships among Staphylococcus species and refinement
} of cluster groups based on multilocus data. BMC Evol. Biol. 12, 171 (2012).

37 Zhou, W. et al. Host-Specific Evolutionary and Transmission Dynamics Shape the Functional Diversification of Staphylococcus epidermidis in Human Skin. Cell 180, 454-470 (2020). 
Lina, G. et al. Bacterial Competition for Human Nasal Cavity Colonization: Role of Staphylococcal agr Alleles. Appl. Environ. Microbiol. 69, 18 (2003).

40 Nagase, N. et al. Isolation and Species Distribution of Staphylococci from Animal and Human Skin. J. Vet. Med. Sci. 64, 245-250 (2002).

41 Wright, J. S. I., Jin, R. \& Novick, R. P. Transient interference with staphylococcal quorum sensing blocks abscess formation. Proc. Natl. Acad. Sci. U. S. A. 102, 1691-1696 (2005).

42 Geisinger, E., Muir, T. W. \& Novick, R. P. agr receptor mutants reveal distinct modes of inhibition by staphylococcal autoinducing peptides. Proc. Natl. Acad. Sci. U. S. A. 106, 12161221, (2009).

43 Nielsen, A. et al. Solonamide B inhibits quorum sensing and reduces Staphylococcus aureus mediated killing of human neutrophils. PLoS One 9, e84992, (2014).

44 Hall, P. R. et al. Nox2 Modification of LDL Is Essential for Optimal Apolipoprotein Bmediated Control of agr Type III Staphylococcus aureus Quorum-sensing. PLoS Pathog. 9, e1003166, (2013).

45 Thompson, J. D., Higgins, D. G. \& Gibson, T. J. CLUSTAL W: improving the sensitivity of progressive multiple sequence alignment through sequence weighting, position-specific gap penalties and weight matrix choice. Nucleic Acids Res. 22, 4673-4680, (1994).

46 Jones, D. T., Taylor, W. R. \& Thornton, J. M. The rapid generation of mutation data matrices from protein sequences. Bioinformatics 8, 275-282, (1992).

47 Vingsbo Lundberg, C. \& Frimodt-Møller, N. Efficacy of topical and systemic antibiotic treatment of meticillin-resistant Staphylococcus aureus in a murine superficial skin wound infection model. Int. J. Antimicrob. Agents 42, 272-275, (2013).

\section{Acknowledgements}

We thank Prof. Horswill (Univerity of Colorado) for his donation of fluorescent reporter strains. We thank Carina Vingsbo Lundberg and Karen Juhl from Statens Serum Institut (DK) for performing the mouse studies under contract. We thank Peter Damborg from Statens Serum Institut (DK) for contributing bacterial isolates. This work was supported by the Danish Independent Research Council-Natural Sciences (Grant No. 0135-00427B; C.A.O.) and the LEO Foundation Open Competition Grant (LF-OC-19-000039; CAO). 


\section{Author contributions}

630 C.A.O. and B.H.G. conceptualised the study; B.H.G., B.S.B., L.V., L.M., and M.S.B. performed the 631 experiments; P.S.A. contributed nasal swap samples and bacterial strains; C.A.O. P.S.A and H.I. 632 supervised the study; B.H.G. and C.A.O. wrote the manuscript with input from all authors; C.A.O. 633 acquired funding.

Competing interests

636 The University of Copenhagen has filed a PCT application (Cysteine derivatives as bacterial 637 antivirulence agents - EP2020/060066) with B.H.G and C.A.O. listed as co-inventors.

640 Supplementary information is available for this paper.

641 Correspondence and requests for materials should be addressed to C.A.O. 


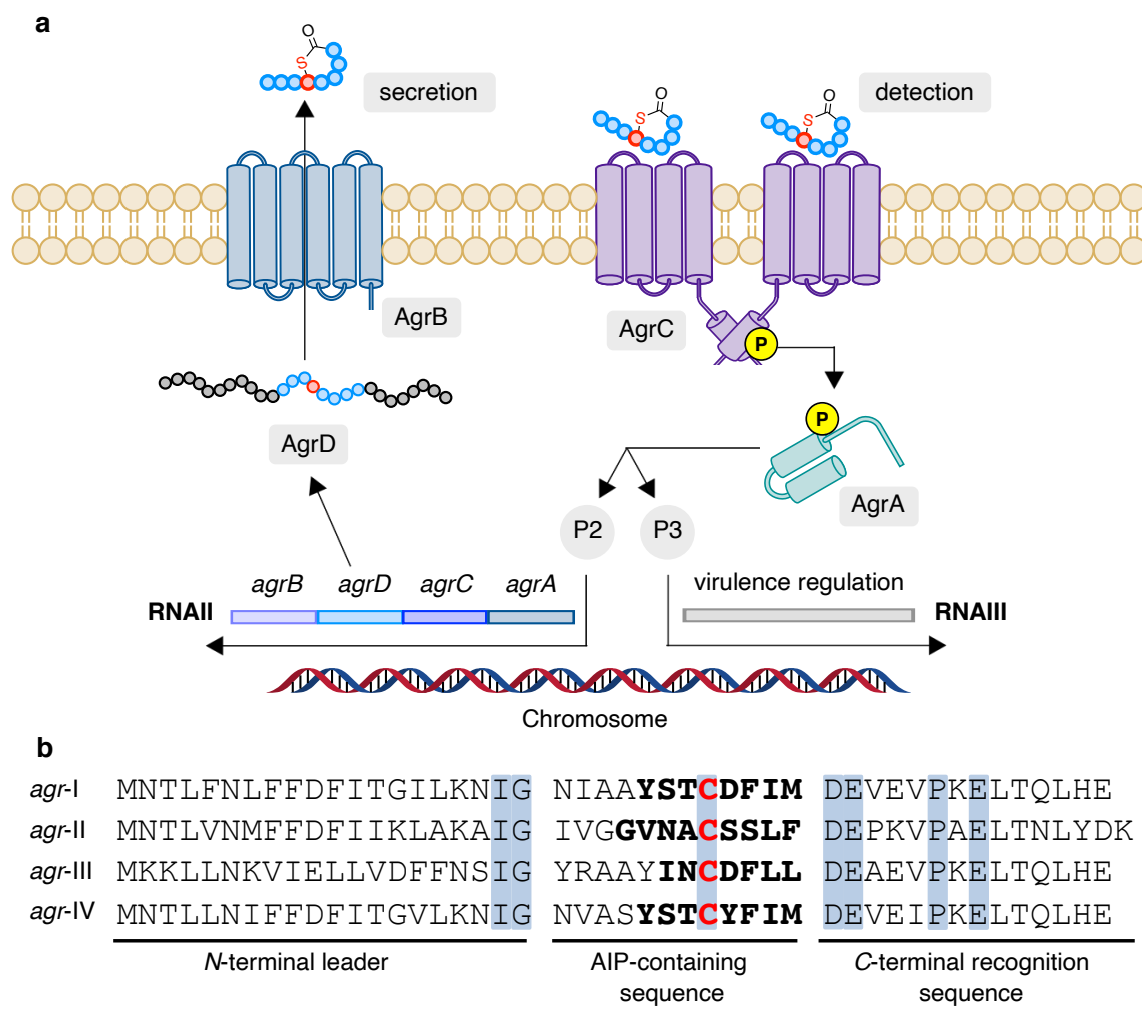

644 Extended Data Fig. 1 | The staphylococcal accessory gene regulator (agr) system. a, The chromosomal agr locus consists of the two promoter regions P2 and P3, which initiate the transcription of RNAII and RNAIII, respectively. RNAII encodes the four protein components (AgrA, AgrB, AgrC, and AgrD) of the agr system, while RNAIII is a regulatory RNA controlling the expression of virulence factors. In the first step of the $a g r$ circuit, the AIP-precursor peptide AgrD (44-46 amino acids for SA) is processed by the membrane-embedded endopeptidase AgrB installing the thiolactone functionality. The peptide is further translocated to the extracellular space and finally cleaved to release the mature AIP. Once a concentration threshold of the AIP molecules is reached due to increased cell density, the AgrC receptor, a homodimeric membrane-bound histidine kinase, is activated. AIP-induced activation is followed by auto-phosphorylation of AgrC and subsequent phosphoryl transfer to AgrA, the response regulator of the agr system, making the AgrC-AgrA interaction a classical two-component regulatory system. Phosphorylated AgrA binds to the promotors $\mathrm{P} 2$ and P3, resulting in upregulated transcription of RNAII and RNAIII, which lead to a positive feedback loop for AgrBDCA expression as well as upregulated expression of virulence factors. $\mathbf{b}$, AgrD peptides consist of three domains, the $C$-terminal recognition sequence, the $N$-terminal leader peptide and in between the 12 amino acid long AIP-containing sequence. Conserved residues are highlighted in blue boxes. 
a

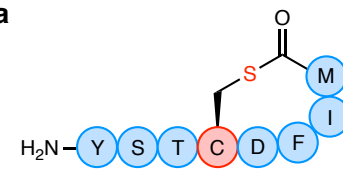

S. aureus AIP-I (1) [S. argenteus AIP-I]

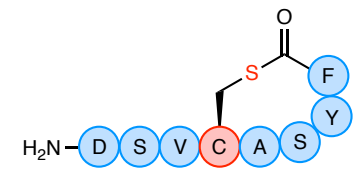

S. epidermidis AIP-I (5)

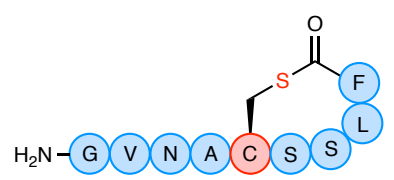

S. aureus AIP-II (2)<smiles>Nc1ccccc1OC1CSC(=O)OCC1O</smiles>

S. aureus AIP-III (3)<smiles>Nc1ccccc1OC1CCC(=O)OC1</smiles>

S. aureus AIP-IV (4) [S. schweitzeri AIP-I]

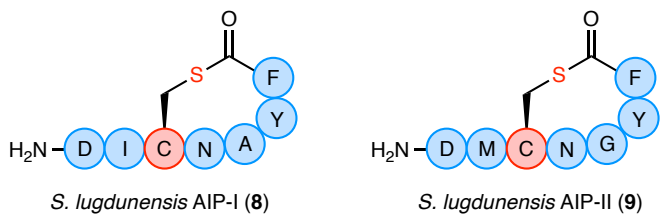<smiles>Nc1ccc(O)cc1</smiles>

S. hominis AIP-I (10)<smiles>Nc1ccc(Oc2ccccc2)cc1</smiles>

S. epidermidis AIP-III (7)

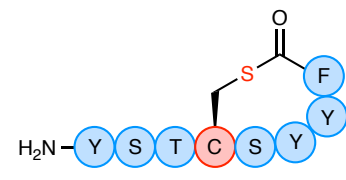

S. caprae AIP-I (12)

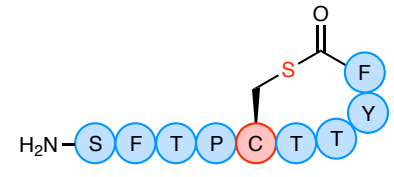

S. haemolyticus AIP-I (13)

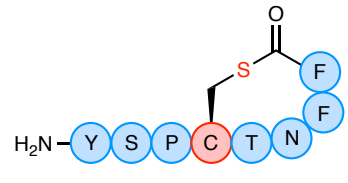

S. warneri AIP-I (14)<smiles>Nc1ccc(OCC(=O)O)cc1</smiles>

b

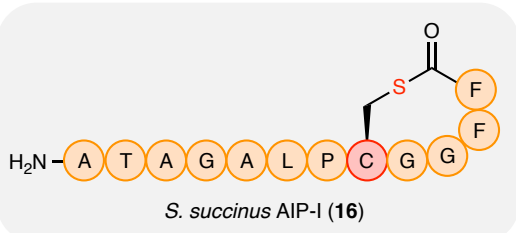

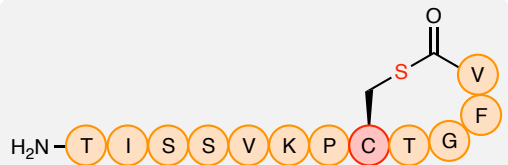

S. cohnii AIP-I (17)

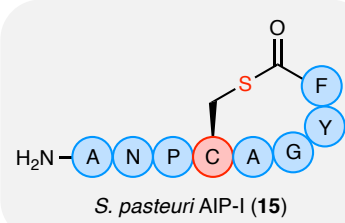

c

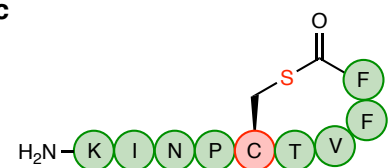

S. hyicus AIP-I (19)

d

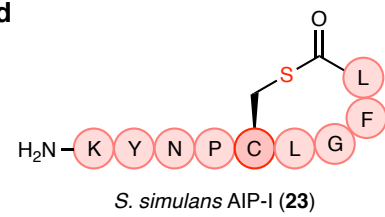

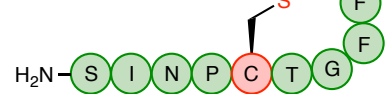

S. chromogenes AIP-I (20)

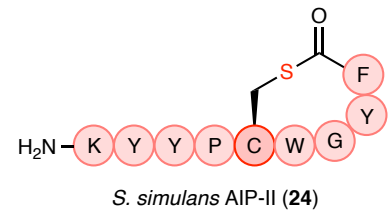

Extended Data Fig. 2 | Structures of the 26 identified AIPs studied herein. a, 15 (17) AIPs known for the Epidermidis-Aureus species group [S. aureus AIP-I-IV (1-4), S. epidermidis AIP-I-III (5-7), S. lugdunensis AIP-I-II (8-9), S. hominis AIP-I-II (10-11), S. caprae AIP-I (12), S. haemolyticus AIP-I (13), S. warneri AIPI (14), S. pasteuri AIP-I (15)]. b, 3 AIPs known from the Saprophyticus species group [S. succinus AIP-I (16), S. cohnii AIP-I (17), S. saprophyticus AIP-I (18)]. c, 4 AIPs known from the Hyicus-Intermedius species group [S. hyicus AIP-I (19), S. chromogenes AIP-I (20), S. schleiferi AIP-I (21), S. intermedius AIP-I (22)]. d, 3 AIPs known from the Simulans species group [S. simulans AIP-I-III (23-25)]. e, 1 AIP known from the Sciuri species group [S. vitulinus AIP-I (26)]; and no AIPs have been identified from Auricularis. AIPs highlighted in grey boxes were newly identified in this study. 
673

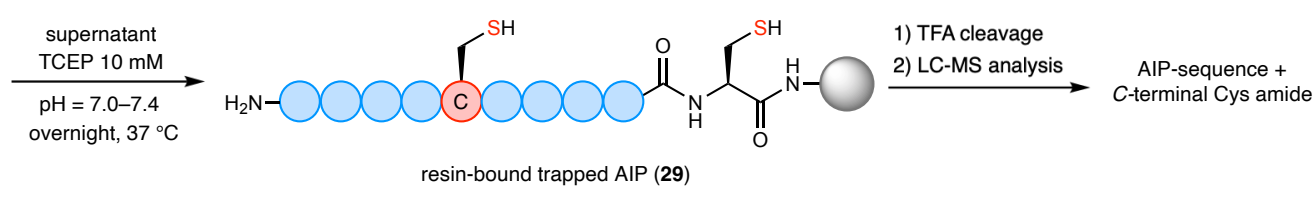

supernatant
$\mathrm{pH}=7.0-7.4$

overnight, $37^{\circ} \mathrm{C}$

resin-bound trapped AIP (29)

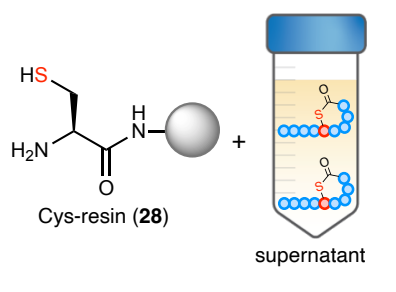

\section{Extended Data Fig. 3 | Identification of AIPs through native chemical ligation (NCL) trapping. Cys-resin}

(28) is incubated overnight in pH-adjusted bacterial supernatant containing tris(2-carboxyethyl)phosphine (TCEP, $10 \mathrm{mM}$ ) enabling chemoselective trapping of AIPs. The resin with the trapped AIP (29) is washed extensively and subsequently treated with trifluoroacetic acid (TFA) to release the trapped AIP. The concentrated cleavage solution is analyzed by liquid-chromatography mass-spectrometry (LC-MS) for the 7 possible AIPs sequences with an additional $C$-terminal Cys amide extracted from the AIP-precursor peptide AgrD. 
a

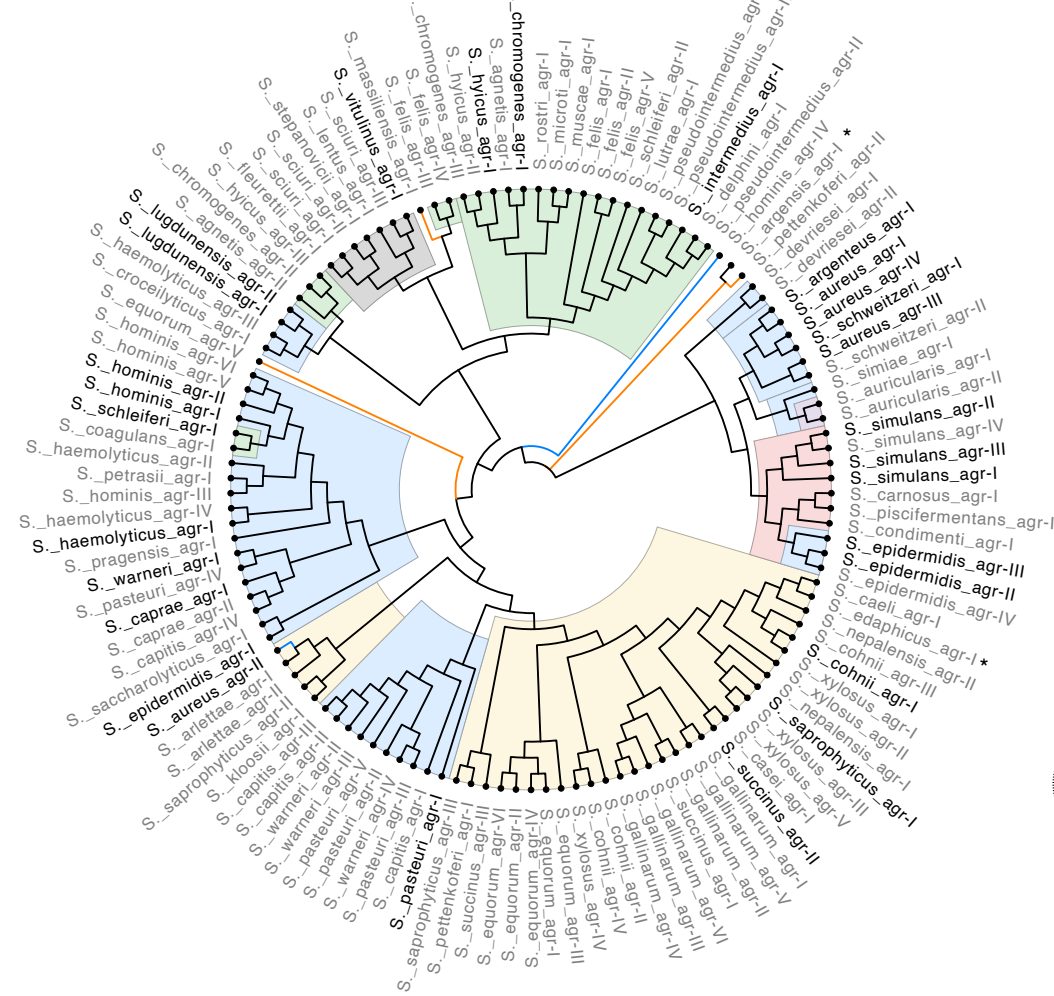

b

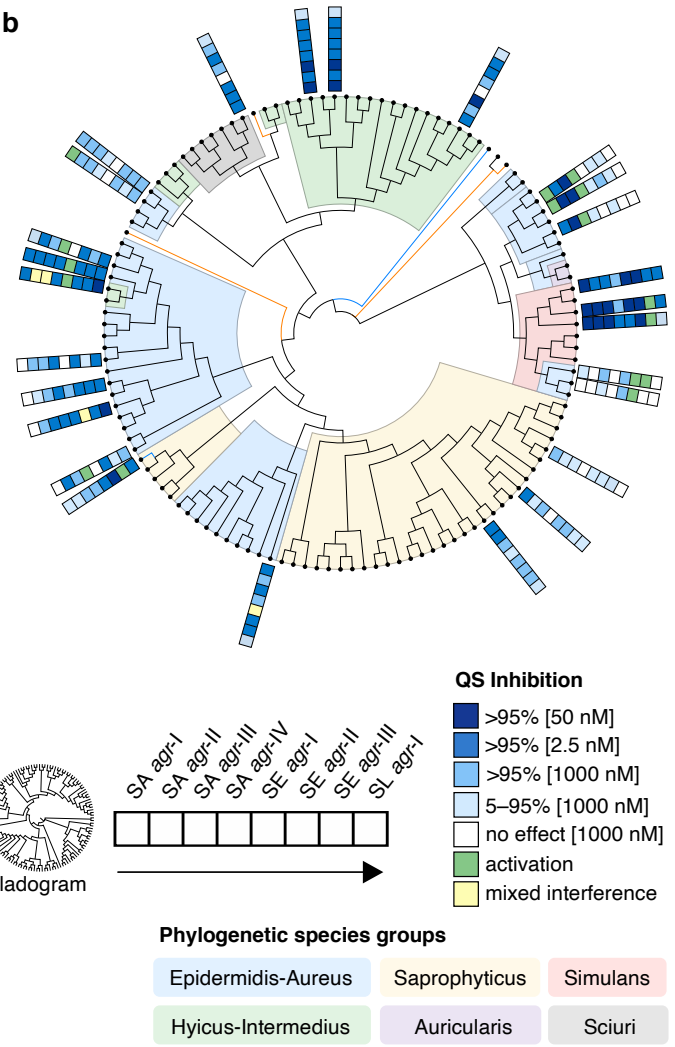

Extended Data Fig. 4 | Phylogeny based on the 12 amino acid AIP-containing sequences. Maximum likelihood tree (bootstrap $n=500$ ) created from 125 AIP-containing sequences extracted from AgrD sequences. The agr groups of identified AIPs were named in their chronological order of their discovery and agr groups with so far unknown AIPs were ranked in order of their number of accessions in the Protein Database. Names of agr groups with known AIPs are black and grey with unknown AIPs. Phylogenetic species groups as defined by Lamers et al. $^{36}$ are colored as follows: Epidermidis-Aureus, blue; Saprophyticus, tan; Simulans, red; Hyicus-Intermedius, green; Auicularis, purple; Sciuri, gray. *Species not yet assigned to species group. b, Cladogram overlaid with QS interactions of known AIPs against SA agr-I-IV, SE agr-I-III and SL agr-I. The interactions of one AIP are represented as colored boxes with SA agr-I closest to the cladogram as depicted in the figure. 
bioRxiv preprint doi: https://doi.org/10.1101/2021.04.18.440348; this version posted April 19, 2021. The copyright holder for this preprint (which was not certified by peer review) is the author/funder, who has granted bioRxiv a license to display the preprint in perpetuity. It is made available under aCC-BY-NC-ND 4.0 International license.

Extended Data Table $1 \mid \mathrm{IC}_{50}$ and $\mathrm{EC}_{50}$ values (nM) measured in $\beta$-lactamase assay using $S$. aureus (SA) AgrC-I-IV reporter strains.

\begin{tabular}{|c|c|c|c|c|}
\hline Species AIP & SA AgrC-I & SA AgrC-II & SA AgrC-III & SA AgrC-IV \\
\hline S. aureus AIP-I (1) & EC50: $5.4 \pm 0.5$ & $700 \pm 165$ & $80 \pm 11$ & EC50: $790 \pm 66$ \\
\hline S. aureus AIP-II (2) & $110 \pm 12$ & EC50: $3.7 \pm 0.5$ & $40 \pm 15$ & $230 \pm 19$ \\
\hline S. aureus AIP-III (3) & $120 \pm 15$ & $150 \pm 63$ & EC50: $11 \pm 1$ & $>1000$ \\
\hline S. aureus AIP-IV (4) & EC50: $59 \pm 2$ & $47 \pm 9 \mathrm{nM}$ & $9.8 \pm 0.2^{a}$ & EC50: $2.6 \pm 0.2$ \\
\hline S. epidermidis AIP-I (5) & $>1000$ & $-b$ & $>1000$ & $-b$ \\
\hline S. epidermidis AIP-II (6) & - & $-b$ & $-b$ & $-b$ \\
\hline S. epidermidis AIP-III (7) & $>10000$ & $-b$ & $>10000$ & $-b$ \\
\hline S. lugdunensis AIP-I (8) & $>1000$ & $-b$ & $>1000$ & $-b$ \\
\hline S. Iugdunensis AIP-II (9) & $>1000$ & $-b$ & $-b$ & $-b$ \\
\hline S. hominis AIP-I (10) & $134 \pm 8$ & $580 \pm 61$ & $220 \pm 46^{a}$ & EC50: $54 \pm 5$ \\
\hline S. hominis AIP-II (11) & $224 \pm 37$ & $>1000$ & $338 \pm 83$ & $-b$ \\
\hline S. caprae AIP-I (12) & $5.7 \pm 0.8$ & $252 \pm 44$ & $-a$ & $199 \pm 19$ \\
\hline S. haemolyticus AIP-I (13) & $340 \pm 25$ & $-b$ & $340 \pm 91$ & - \\
\hline S. warneri AIP-I (14) & $100 \pm 10$ & $1000 \pm 105$ & $460 \pm 59$ & $>1000$ \\
\hline S. pasteuri AIP-I (15) & $155 \pm 34$ & $-b$ & $191 \pm 18$ & $>1000$ \\
\hline S. succinus AIP-I (16) & $117 \pm 26$ & $854 \pm 65$ & $>1000$ & $>1000$ \\
\hline S. cohnii AIP-I (17) & $>10000$ & $-b$ & $-b$ & $-b$ \\
\hline S. saprophyticus AIP-I (18) & $360 \pm 30$ & - & $-b$ & $-b$ \\
\hline S. hyicus AIP-I (19) & $3.3 \pm 0.5$ & $350 \pm 90$ & $4.0 \pm 0.8$ & $180 \pm 33$ \\
\hline S. chromogenes AIP-I (20) & $15 \pm 1$ & $200 \pm 13$ & $60 \pm 13$ & $350 \pm 85$ \\
\hline S. schleiferi AIP I (21) & $2.8 \pm 0.8$ & $86 \pm 6$ & $80 \pm 16^{a}$ & EC50: $31 \pm 6$ \\
\hline S. intermedius AIP-I (22) & $87 \pm 20$ & $>1000$ & $104 \pm 20$ & $-b$ \\
\hline S. simulans AIP-I (23) & $8.6 \pm 0.5$ & $23 \pm 2$ & $50 \pm 11$ & $280 \pm 66$ \\
\hline S. simulans AIP-II (24) & $1.9 \pm 0.3$ & $80 \pm 7$ & $198 \pm 33$ & $273 \pm 26$ \\
\hline S. simulans AIP-III (25) & $1.6 \pm 0.1$ & $60 \pm 7$ & $103 \pm 5$ & $351 \pm 60$ \\
\hline S. vitulinus AIP-I (26) & $190 \pm 15$ & $800 \pm 116$ & $690 \pm 46$ & $-b$ \\
\hline S. aureus AIP-III D4A (29) & $3.3 \pm 0.7$ & $34 \pm 1$ & $1.6 \pm 0.2^{a}$ & $10 \pm 0.2$ \\
\hline
\end{tabular}

All inhibition assays were performed in the presence of $100 \mathrm{nM}$ cognate AIP and the results represent means \pm standard error of the mean (SEM) of at least duplicate determinations performed in biological triplicate.

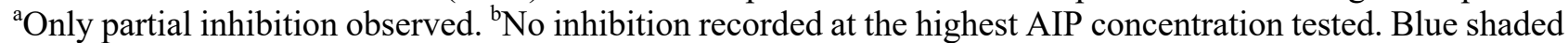
boxes represent $\mathrm{IC}_{50}$ values determined in this study. White represent $\mathrm{IC}_{50} / \mathrm{EC}_{50}$ values determined previously ${ }^{34}$. The AIPs of SE (5-7) and SL-AIP-I (8) were weak inhibitors or displayed no significant inhibition at the highest tested concentration. The $S$. hominis AIP-II (11) was a moderate inhibitor of SA $a g r$-I and $a g r$-III with lower potency against agr-II and no effect on agr-IV. The $S$. caprae AIP-I (12) displayed high potencies against $a g r$-I and $a g r$-II and moderate potency against $a g r$-IV. The AIP was not able to inhibit $a g r$-III at higher concentrations but showed inhibtion of agr activity at lower nanomolar concentrations. The S. pasteuri AIP-I (15) and $S$. succinus AIP-I (16) exhibited moderate to low potencies against the four reporter strains, while the S. cohnii AIP-I (17) showed only limited inhibition of agr-I. The $S$. intermedius AIP (22) exhibited moderate and weak potency against $a g r$-I and $a g r$-II, respectively. Higher potency was observed against $a g r$-III and no effect was seen against agr-IV. S. simulans AIP-II-III $(\mathbf{2 4}, \mathbf{2 5})$ were highly potent inhibitors of $a g r$-I and $a g r-$ II and exhibited slightly decreased potencies against agr-III and moderate inhibition against agr-IV. 
bioRxiv preprint doi: https://doi.org/10.1101/2021.04.18.440348; this version posted April 19, 2021. The copyright holder for this preprint (which was not certified by peer review) is the author/funder, who has granted bioRxiv a license to display the preprint in perpetuity. It is made available under aCC-BY-NC-ND 4.0 International license.

712 Extended Data Table 2 | IC I $_{50}$ values (nM) determined using fluorescent reporter strains of S. aureus (SA) 713 agr-I-IV, S. epidermidis (SE) agr-I-III and S. lugdunensis (SL) agr-I.

\begin{tabular}{|l|c|c|c|c|c|c|c|}
\hline $\begin{array}{c}\text { Reporter } \\
\text { strain }\end{array}$ & $\begin{array}{c}\text { S. aureus } \\
\text { AIP-I (1) }\end{array}$ & $\begin{array}{c}\text { S. epidermidis } \\
\text { AIP-I (5) }\end{array}$ & $\begin{array}{c}\text { S. } \\
\text { lugdunensis } \\
\text { AIP-I (8) }\end{array}$ & $\begin{array}{c}\text { S. simulans } \\
\text { AIP-I (23) }\end{array}$ & $\begin{array}{c}\text { S. simulans } \\
\text { AIP-II (24) }\end{array}$ & $\begin{array}{c}\text { S. simulans } \\
\text { AIP-III (25) }\end{array}$ & $\begin{array}{c}\text { S. aureus } \\
\text { AIP-III D4A } \\
(\mathbf{2 7})\end{array}$ \\
\hline SA agr-I & act. & $67 \pm 16$ & $138 \pm 26$ & $0.34 \pm 0.02$ & $0.45 \pm 0.03$ & $0.18 \pm 0.03$ & $0.46 \pm 0.05$ \\
\hline SA agr-II & $5.8 \pm 0.7$ & $858 \pm 236$ & $234 \pm 17$ & $0.31 \pm 0.01$ & $0.8 \pm 0.2$ & $0.22 \pm 0.06$ & $0.36 \pm 0.01$ \\
\hline SA agr-III & $0.91 \pm$ & $3.5 \pm 0.2$ & $22 \pm 2$ & $0.53 \pm 0.02$ & $2.8 \pm 0.3$ & $0.59 \pm 0.04$ & $0.16 \pm 0.01$ \\
\hline $\begin{array}{l}0.05 \\
\text { IV agr- }\end{array}$ & act. & - & - & $10.0 \pm 0.5$ & $31 \pm 7$ & $34 \pm 12$ & $0.70 \pm 0.02$ \\
\hline SE agr-I & $>1000$ & act. & $138 \pm 5$ & $0.74 \pm 0.01$ & $0.31 \pm 0.06$ & $0.14 \pm 0.03$ & - \\
\hline SE agr-II & $>1000$ & $10.1 \pm 0.8$ & $11.6 \pm 1.2$ & $0.26 \pm 0.01$ & $0.26 \pm 0.08$ & $0.11 \pm 0.02$ & $424 \pm 27$ \\
\hline SE agr-III & - & $8.6 \pm 0.7$ & $66.8 \pm 2.9$ & act. & $0.6 \pm 0.2$ & act. & - \\
\hline SL agr-I & - & - & act. & $113 \pm 11$ & $1.2 \pm 0.2$ & $0.48 \pm 0.06$ & - \\
\hline
\end{tabular}

$714 \quad \mathrm{IC}_{50}$ values represent means \pm standard error of the mean (SEM) of at least duplicate determinations performed 715 in biological triplicate. act. denotes activation. 